\title{
Evolutionary dynamics driving continental radiations of Fagaceae forests across the Northern Hemisphere
}

\section{Biao-Feng Zhou}

South China Botanical Garden, Chinese Academy of Sciences

\section{Shuai Yuan}

South China Botanical Garden, Chinese Academy of Sciences

\section{Andrew Crowl}

Department of Biology, Duke University

\section{Yi-Ye Liang}

South China Botanical Garden, Chinese Academy of Sciences

\section{Yong Shi}

South China Botanical Garden, Chinese Academy of Sciences

\section{Xue-Yan Chen}

South China Botanical Garden, Chinese Academy of Sciences

\section{Qing-Qing An}

South China Botanical Garden, Chinese Academy of Sciences

\section{Ming Kang}

South China Botanical Garden, Chinese Academy of Sciences https://orcid.org/0000-0002-4326-7210

\section{Paul Manos}

Department of Biology, Duke University

\section{Baosheng Wang ( $\sim$ baosheng.wang@scbg.ac.cn)}

South China Botanical Garden, Key Laboratory of Plant Resources Conservation and Sustainable Utilization, Chinese Academy of Sciences

\section{Article}

Keywords: macroevolutionary events, genome-wide signatures, hybridization

Posted Date: October 26th, 2021

DOl: https://doi.org/10.21203/rs.3.rs-968321/v1

License: (c) (1) This work is licensed under a Creative Commons Attribution 4.0 International License. 
Version of Record: A version of this preprint was published at Nature Communications on March 14th, 2022. See the published version at https://doi.org/10.1038/s41467-022-28917-1. 
1 Evolutionary dynamics driving continental radiations of Fagaceae forests across

2 the Northern Hemisphere

3

4 Biao-Feng Zhou ${ }^{1,2,5 ;}$ ORCID: 0000-0002-2782-4160

5 Shuai Yuan ${ }^{1,5}$; ORCID: 0000-0002-9851-6384

6 Andrew A. Crowl 3 3; ORCID: 0000-0002-1745-0687

7 Yi-Ye Liang 1; ORCID: 0000-0002-4992-3784

8 Yong $\mathrm{Shi}^{1}$

9 Xue-Yan Chen ${ }^{1}$

10 Qing-Qing $\mathrm{An}^{1}$

11 Ming Kang 1,4; ORCID: 0000-0002-4326-7210

12 Paul S. Manos ${ }^{3 *}$; ORCID: 0000-0003-3122-1538

13 Baosheng Wang ${ }^{1,4^{*}}$; ORCID: 0000-0002-0934-1659

Affiliations:

$16{ }^{1}$ Key Laboratory of Plant Resources Conservation and Sustainable Utilization, South

17 China Botanical Garden, Chinese Academy of Sciences, Guangzhou 510650,

18 China

$19{ }^{2}$ University of the Chinese Academy of Sciences, Beijing 100049, China

$20{ }^{3}$ Department of Biology, Duke University, Durham, NC 27708, USA

$21{ }^{4}$ Center of Conservation Biology, Core Botanical Gardens, Chinese Academy of

22 Sciences, Guangzhou 510650, China

$24{ }^{5}$ These authors contributed equally to this work

25 *Corresponding authors: pmanos@duke.edu, baosheng.wang@scbg.ac.cn 


\section{Introductory paragraph}

36 Northern Hemisphere forests changed drastically in the early Eocene with the 37 diversification of the oak family (Fagaceae). Cooling climates over the next 20

38 million years fostered the spread of temperate biomes that became increasingly 39 dominated by oaks and their chestnut relatives. Here we investigate the timing and 40 pattern of major macroevolutionary events and ancient genome-wide signatures of 41 hybridization across Fagaceae. An unparalleled transformation of forest dynamics 42 began with the rapid diversification of major lineages within 15 million years 43 following the K-Pg extinction. Innovations related to seed and pollen dispersal are 44 implicated in triggering waves of continental radiations, while fungal symbioses 45 fortified a competitive edge underground. We detected introgression at multiple time 46 scales, including ancient events predating the origination of genus-level diversity. As 47 oak lineages moved into newly available temperate habitats in the early Miocene, 48 secondary contact between previously isolated species occurred. This resulted in 49 adaptive introgression, further amplifying global proliferation.

\section{Main text}

52 Northern Hemisphere forests and shrublands are now dominated by species 53 comprising temperate and subtropical lineages, marking one of the greatest floristic 54 transitions in the vegetation history of the Cenozoic ${ }^{1-3}$. Paleobotanical reconstructions 55 suggest that a cooling global climate afforded ecological opportunities to plant groups 56 that were physiologically predisposed to disperse into and radiate within broadening 57 and often repeated seasonal biomes across what would become the Americas and 58 Eurasia $^{4-7}$. Central to this pattern of floristic replacement with significant ecological 59 consequence are the roughly 900 species currently recognized within Fagaceae (oak, 60 beech, chestnut, stone oak). Important components of the timing and pattern of 61 macroevolutionary events and the role of ancient hybridization, however, have yet to 62 be sufficiently described across Fagaceae.

63 The oak family plays a major ecological role in terms of sheer abundance of 64 standing biomass ${ }^{6,8-16}$ and a variety of mutualistic associations involving 65 ectomycorrhizal fungi ${ }^{17-19}$, gall-forming insects ${ }^{20-22}$, and seed-dispersing vertebrates ${ }^{23-}$ 6628 . Interactions between Fagaceae and their co-distributed biota suggests degrees of 67 host specificity and the potential for co-evolution, reciprocal diversification, and 68 expansion of range size. 
Fossil analogs of modern Fagaceae are well represented in the Northern

70 Hemisphere, indicating long-term presence and differential patterns of 71 diversification ${ }^{29-37}$. Recent studies integrating these fossils within phylogenies of 72 modern taxa have provided essential context to estimate divergence times ${ }^{38-40}$. With a 73 minimum age of ca. 80 million years ago (Ma) and precise aging of new fossilized 74 pollen and macrofossils assigned to some modern groups by $50 \mathrm{Ma}$, the evolution of 75 major lineages appears to be unusually rapid for forest tree species ${ }^{41,42}$. However, the 76 diversification history of Fagaceae remains incompletely understood, with the 77 exception of modern lineages of Quercus ${ }^{39,43-45}$. Therefore, a complete historical 78 account of this continental radiation is needed to bring to light the dynamics of speciation through the genomes of these ecologically important tree species.

Oaks have a long history of divergence in spite of gene flow. Recent estimates

81 of phylogeny using next generation sequencing of nuclear DNA resolve the main oak 82 groups while demonstrating that oak species are generally not of hybrid origin ${ }^{39,46}$. 83 However, more targeted phylogenomic studies have shown that hybridization has left 84 its signature: one of unstable lineages and taxa, the likely result of ancient 85 hybridization, another of intermediate position between parental lineages as expected 86 by recent-generation hybrids ${ }^{47-50}$. New insights into nuclear genomic architecture of 87 hybridization complement various datasets derived from the maternally-inherited 88 plastome and suspected cases of plastome capture and resulting cytoplasmic-nuclear 89 discordance have been shown at various phylogenetic depths in Quercus $^{51-53}$. Now the 90 timing and impact of these events within Quercus, as well as within and between 91 other lineages, is within reach: chronograms for both genomes along with thorough 92 interrogation of the nuclear genome using modern analytical approaches provides the 93 framework needed to estimate the timing of hybridization events, identify the 94 signatures of gene flow, and detect evidence for adaptive evolution.

95 Phylogenomic analyses of nuclear and plastid genomes reveal a complex 96 history of divergence and gene flow in deep time across Fagaceae. To test specific 97 hypotheses of ancient hybridization, we constructed time-calibrated phylogenies to 98 pinpoint major divergent and reticulate events across a broad sample of 122 99 individual plants representing 91 species from all recognized genera, using 2,124 100 nuclear loci and full plastomes (Supplementary Tables 1 and 2). With these data, we 101 characterize the diversification of Fagaceae and identify admixed genomes due to 102 ancient gene flow within the first complete family-wide phylogenetic context. 


\section{Results and Discussion}

\section{Time-calibrated phylogeny based on nuclear data}

105 Maximum likelihood (ML) and Bayesian analyses of the concatenated dataset and 106 coalescent analyses using ASTRAL-III and SVDquartets produced similar trees with 107 strong support (BS $>90$ and $\mathrm{BI}>0.95$ ) for all nodes except a few branches (Fig. 1;

108 Supplementary Fig. 1). All genera of Fagaceae were inferred to be monophyletic with 109 fully resolved interrelationships. Our phylogenetic estimate unambiguously supports 110 three early-diverging lineages of Fagaceae - Fagus, Trigonobalanus, and two 111 castaneoid lineages, Castanea + Castanopsis - along with a novel resolution for a 112 crown clade comprising the three remaining castaneoid genera, Chrysolepis, 113 Lithocarpus, and Notholithocarpus, which in turn is sister to Quercus (Fig. 1; 114 Supplementary Fig. 1). Resolution of castanoid taxa (Chrysolepis, Lithocarpus, and 115 Notholithocarpus) as sister to Quercus settles long-standing questions on the origin of 116 the wind-pollinated oaks: they are derived from insect-pollinated ancestors that 117 already possessed single rounded fruit seated within a valveless cupule ${ }^{54}$. Within 118 Quercus, our analyses confirmed the phylogenetic structure resolved by previous 119 studies based on sequences derived from RAD-seq datasets ${ }^{39,50}$ and nuclear loci ${ }^{47}$. 120 Despite phylogenetic congruence across methods, high levels of gene-tree conflict 121 within the nuclear genome were observed, likely due to incomplete lineage sorting 122 (ILS; Supplementary Figs. 2, 3 and 4). This would be expected given the rapid 123 evolution of crown clade genera as inferred here (see below).

124 We constrained nodes with eight fossil calibrations (Fig. 1; Supplementary 125 Table 3) to estimate divergence times and diversification dynamics within Fagaceae.

126 Two early-diverging lineages of Fagaceae originated by the late Cretaceous, with 127 Fagus and Trigonobalanus diverging at $82.6 \mathrm{Ma}(95 \% \mathrm{CI}=84.2-80.1 \mathrm{Ma})$ and 70.7 $128 \mathrm{Ma}(95 \% \mathrm{CI}=69.1-62.4 \mathrm{Ma})$, respectively (Fig. 1). Subsequent branching events in 129 the early Cenozoic suggest that the six genera (Castanea, Castanopsis, Chrysolepis, 130 Lithocarpus, Notholithocarpus and Quercus; the hypogeous seed or "HS" clade 131 hereafter $)$ that comprise $98.8 \%(N=893)$ of the modern species originated during the 132 Paleocene. The ancestor of the HS clade split at $64.5 \mathrm{Ma}(95 \% \mathrm{CI}=69.1-62.4 \mathrm{Ma})$ 133 followed by the rapid origination of extant genera within a $15 \mathrm{Ma}$ window (Fig. 1). 134 These events closely follow the Cretaceous-Paleogene (K-Pg) boundary dated at 66 $135 \mathrm{Ma}^{55}$. 
Accelerated diversification following the K-Pg mass extinction event has been documented in plants ${ }^{56,57}, \operatorname{birds}^{58}$, frogs $^{59}$, fish ${ }^{60}$, and mammals ${ }^{61}$, most likely a

138 generalized consequence of ecological opportunities following the mass extinction.

139 An increase in speciation rate just after the K-Pg boundary was confirmed for

140 Fagaceae by diversification rate analyses, with a net speciation rate shift detected

141 along the branch leading to the ancestor of the HS clade (Fig. 1). This result is robust

142 to different calibration sets and reference trees for molecular dating (Supplementary

143 Fig. 5).

\section{Ecological correlates of diversification}

146 The HS clade shares the derived feature of hypogeous germination, as defined by the

147 first leaves of the embryo remaining in the seed as storage organs that contribute to

148 enhancing seedling survivorship ${ }^{26}$. This condition is often correlated with larger seeds

149 that are biotically dispersed by various specialized animal groups whereas the two

150 early-branching lineages (Fagus and Trigonobalanus) share the plesiomorphic

151 condition of smaller seeds and the generalized state of epigeal germination ${ }^{62}$. Previous

152 phylogenetic studies including fossils have revealed several transitions to biotic

153 dispersal across fagalean lineages during its ca. 95 million-year history ${ }^{63,64}$. Biotically

154 dispersed lineages have larger range sizes and higher diversification rates than

155 abiotically dispersed lineages. Innovations associated with seed morphology coincide

156 with an increase in diversification rate of the HS clade after the K-Pg boundary (Fig.

157 1). Time-calibrated phylogenies of the main groups of modern HS seed dispersers,

158 specifically scatter-hoarding Sciuridae (squirrels), Covidae (jays), and Picidae

159 (woodpeckers) contrast sharply. Evolution of rodent-mediated dispersal closely

160 follows the origin of the HS clade and other large-seeded biotically dispersed fagalean

161 lineages supporting a generalized co-evolution with early-diverging Sciuridae ${ }^{61,65-67}$.

162 In contrast, the relative timing for the diversification of bird lineages associated with

163 the dispersal of HS seed is at least 20 million years later ${ }^{68,69}$. This suggests a second

164 phase of mutualistic response driven by HS seed production generated patterns of co-

165 distribution between granivorous birds, best exemplified in Quercus, that likely began

166 during the Miocene $\mathrm{M}^{24,70}$.

167 Ecological success of Fagaceae is often attributed to symbiosis with at least 168 three main ectomycorrhizal (ECM) lineages of basidiomycetes: Russulales, Boletales, 169 and Agaricales $^{71}$. This mutualism represents an ancient resource-sharing mechanism 
170 that contributes heavily to ecosystem processes and dominance of Fagaceae ${ }^{17,72,73}$.

171 The estimated number of global ECM fungal species is c. 6,000 with Fagaceae 172 accounting for $45 \%$ of the associated 2,000 species of host seed plant diversity ${ }^{71}$. 173 While the stem lineages of the main ECM clades date back to the Jurassic, crown 174 clade diversification and inferred shifts in speciation rate occur contemporaneously in 175 many of the lineages associated with Fagaceae ${ }^{74}$. Multiple increases in speciation rate 176 postdate the K-Pg boundary by at least 20 million years, suggesting that transition to 177 Fagaceae forests in the Oligocene may have contributed to species radiations linked to 178 symbiosis. Indeed, secondary increases in speciation rate spanning the Oligocene and 179 Miocene were detected in three clades, Lithocarpus from southeast Asia, the Eurasian 180 subclade of section Quercus, and section Lobatae which is endemic to the Americas 181 (Fig. 1; Supplementary Fig. 5). Previous studies based on global sampling of oak 182 species reported four shifts of diversification during the Miocene ${ }^{39}$, including the two 183 events we observed within Quercus.

184 Rapid radiation of the genus Quercus is consistent with global temperature 185 cooling associated with the onset of temperate habitats during the Oligocene (Fig. 186 1A). Our phylogenetic analyses confirm that Quercus evolved from within a clade 187 formed by all five insect-pollinated castaneoid genera, and diverged from them 188 approximately $56 \mathrm{Ma}$ (Fig 1; Supplementary Fig. 5). Fossilized pollen assignable to 189 modern oak sections is found at high latitudes well before Quercus migrated to 190 middle latitudes ${ }^{32}$. Thus, the origin of wind-pollination in Quercus preceded the 191 explosive radiations of oaks in the Oligocene to early Miocene. Shift to wind192 pollination alone did not increase the diversification rate of oak species immediately, 193 but instead served as a predisposed neutral change that later facilitated rapid radiation 194 of this genus during the expansion of seasonal climates (Fig. 1). Consistent with this 195 expectation, oaks have their highest species richness in cool-temperate areas in 196 middle latitudes and montane areas at lower latitudes of the Americas, where they 197 form ecologically dominant forests ${ }^{6,46}$.

\section{Ancient hybridization explains cytoplasmic-nuclear gene tree conflict}

200 Plastome-based analyses using various phylogenetic methods (ML or BI analyses), 201 data partitioning schemes (un-partitioned or partitioned by gene and codon position), 202 and alignments (nucleotide or amino acid sequences) yielded largely congruent 203 topologies (Supplementary Fig. 6). Major nodes along the backbone of the plastid tree 
204 were highly supported (BS $>80 \%$ and $\mathrm{BI}>95 \%$ ) and consistent with the nuclear 205 trees in the resolution of Fagus and Trigonobalanus as early-branching lineages (Fig.

206 2). The plastome topology, however, differs markedly from the trees obtained with 207 nuclear loci in regards to the composition and placement of major lineages within the 208 HS clade (Fig. 2). While most plastome subclades comprise related species, several 209 combine disparate taxonomic groups. We failed to recover monophyly of two genera, 210 Quercus and Notholithocarpus, and five sections of Quercus (Quercus, Virentes,

211 Ponticae, Protobalanus, Ilex and Cerris). The structure of the plastome reconstruction 212 within the HS clade is largely geographic, consistent with previous studies ${ }^{52,53,75,76}$, 213 with the taxonomic diversity divided into two major clades we treat here as New 214 World (NW) and Old World (OW) (Fig. 2).

215 The NW-OW pattern recovered in our plastome analyses suggests an early 216 geographic homogenization of cytoplasm across lineages generating the observed 217 cytoplasmic-nuclear discordance at the deepest level of the HS clade (Fig. 2). While 218 the most likely source of cytoplasmic-nuclear discordance is hybridization, 219 incomplete lineage sorting (ILS) could produce a similar pattern. To discriminate 220 between these two hypotheses, we performed coalescent-based simulations. We found 221 the plastid tree discordance to be significantly higher than the expected distribution 222 under a strict coalescent process (Supplementary Fig. 7) and conflicting plastid 223 bipartition frequencies at or near zero in the 10,000 simulated organellar gene trees 224 (Supplementary Fig. 8). ILS alone is therefore insufficient to explain the observed 225 cytoplasmic-nuclear incongruence recovered in these datasets and a scenario of 226 historical gene flow must be invoked.

227 Hybridization is a widespread phenomenon within modern lineages of 228 Fagaceae, especially between species within sections of Quercus $^{77}$, and plastome 229 capture events are well documented between sympatric species ${ }^{52,78-80}$. Hybridization 230 is also prevalent between closely related species across many genera within other 231 fagalean families ${ }^{81-83}$. However, gene flow between modern genera is without 232 precedent. When we applied molecular dating methods to the full plastome data, we 233 found estimated divergence times for the deepest splits to generally fall within the 234 rapid diversification phase for the HS clade based on nuclear data (Fig. 1; 235 Supplementary Fig. 9). Without invoking non-sexual processes such as transmission 236 between incompatible species through intimate physical contact, e.g., plant-plant 237 parasitism and natural root grafts ${ }^{84}$, ancient hybridization is the most likely source of 
238 deep cytoplasmic-nuclear conflict in Fagaceae. Taken together, our results indicate

239 this pattern of geographic division of reciprocally monophyletic plastome types is best

240 explained as a vestige of widespread ancient hybridization among ancestral

241 populations of the HS clade that became spatially isolated by paleogeographic barriers

242 to gene flow dated minimally to the early Paleocene (Fig. 2; Supplementary Fig. 9).

243 We additionally found evidence for more recent plastome capture events

244 resulting from hybridization within Quercus between the late Miocene to Pliocene

245 (Fig. 2; Supplementary Fig. 9). As expected, the pattern of discordance between

246 plastome and nuclear genomes uncovers multiple instances where species from

247 phylogenetically distinct clades, but overlapping geographic ranges, share plastome

248 types, for example between species of sections: Ilex and Cerris, Virentes and

249 Quercus, Protobalanus and Quercus, and Ponticae and Quercus (Fig. 2). While

250 inferring ancient hybridization events using cytoplasmic-nuclear gene tree conflict

251 provides some evidence of reticulate evolutionary history, satisfactorily confirming

252 and characterizing ancient gene flow requires a detailed investigation into the nuclear

253 genome.

\section{Ancient gene flow and adaptive introgression detected in the nuclear genome}

256 Extensive investigation using a $D$-statistic (ABBA-BABA) test detected significant 257 gene flow on $236(0.911 \%)$ of 25882 trios extracted from the species tree $(P<0.01$ 258 after Bonferroni correction) (Supplementary Table 4). Not surprisingly, most cases of 259 gene flow appeared to be recent in origin and between closely related species from 260 within genera or sections of Quercus (Fig. 3A). Ancient gene flow, however, was 261 detected between Eurasian white oaks (section Quercus) and Q. pontica (section 262 Ponticae) and between North American white oaks (section Quercus) and the 263 ancestor of section Virentes (Fig. 3), consistent with the results of gene tree analyses 264 from the two genomes (Fig. 2). Network analyses using SNaQ confirmed historical 265 gene flow between $Q$. pontica and Eurasian white oaks inferred in the current study 266 (Supplementary Fig. 10) and previous studies ${ }^{47}$.

267 We also assessed the distribution of alternative topologies within our 2124

268 nuclear gene dataset and found introgressed signals to be widely scattered across the 269 genome (Supplementary Fig. 11). This is expected given that long-term 270 recombination tends to fragment introgressed stretches of DNA following initial 271 hybridization events ${ }^{85}$. However, positive selection has been shown to maintain long 
272 introgressed haplotypes in populations of humans and maize ${ }^{86,87}$, with the length of 273 introgressed fragments increasing with stronger selection ${ }^{88}$. Our investigation of 274 putatively ancient hybridization events between sections of Quercus yielded 275 haplotypes that were significantly longer than expected under neutrality. Identity-by276 descent (IBD) analyses based on whole genome SNP data clearly detected a large 277 number of shared haplotype blocks (see Methods) for three lineage-pairs, i.e., $Q$. 278 pontica vs. European and Asian white oaks, North American white oaks vs. section 279 Virentes, and North American white oaks vs. Q. sadleriana (Fig. 4B-D). However, we 280 did not find IBD blocks of similarly long lengths between other Quercus sections in 281 which we documented plastome capture events (Supplementary Table 5).

282 Within the long sets of shared IBD regions, the $D$-statistic test revealed gene 283 flow between oak sections. In addition, the recombination rate in the same IBD 284 regions was not different from genomic background ( $W$ ranges from 82978 to 321198 , $285 P=0.66-0.73$, Mann-Whitney $U$ test; Supplementary Table 6), and the length of the 286 IBDs was not associated with recombination rate (Spearman's $\rho=-0.19-0.14, P=$ $2870.10-0.79$; Supplementary Fig. 12). Therefore, these haplotypes shared between 288 Quercus sections are most likely due to historical inter-sectional hybridization instead 289 of the maintenance of ancestral polymorphisms in regions with reduced 290 recombination rates.

291 To test this prediction, we calculated the probability of maintaining selectively 292 neutral haplotypes of a given length in both oak sections after introgression using 293 methods developed to study introgression in humans ${ }^{86}$ and using generation times and 294 mutation and recombination rates derived for oak species ${ }^{89-91}$. We determined that 295166 IBD blocks (11724 -113757 bp) were significantly longer than expected if the 296 introgressed fragments were selectively neutral $(P<0.05$; Fig. $4 \mathrm{~d}$; see details in 297 Methods), suggesting that the IBDs identified here provide convincing evidence of 298 adaptive introgression. Multiple GO categories with important metabolic processes 299 and molecular functions (e.g., terpene metabolic processes, sesquiterpenoid metabolic 300 processes) were overrepresented for genes located in these IBD regions 301 (Supplementary Table 7), further suggesting a diverse set of genes and functional 302 categories may have contributed to adaptive introgression of oak species. Adaptive 303 introgression between closely related species has recently been documented in 304 Quercus $^{92}$. Our study posits that introgressed elements between divergent oak sections 305 could be preserved for millions of years by natural selection. 
With the exception of the few cases involving sections Quercus, Ponticae and

307 Virentes, we found no corroborating evidence of hybridization within the nuclear 308 genome of the remaining lineages exhibiting cytoplasmic-nuclear gene tree conflict.

309 The occurrence of plastome capture events in the absence of detectable nuclear 310 introgression is not unexpected, and could be due to the early phases of hybrid zone 311 dynamics $^{93,94}$. For example, extensive backcrossing with one parental species after 312 initial hybridization could sweep out signals of reticulation events in the nuclear 313 genome and recombination over long evolutionary time could have degraded signals 314 of ancient hybridization ${ }^{95,96}$. In oaks, backcrossing is preferentially unidirectional ${ }^{97,98}$ 315 and linkage disequilibrium typically declines to background quickly (within $1 \mathrm{~kb}$ ) ${ }^{99,100}$, 316 blurring the signals of past introgression in the nuclear genome. Alternatively, as 317 mentioned above, plastomes can be captured through non-hybridizing means such as 318 intimate physical contact, e.g. plant-plant parasitism and natural grafts ${ }^{84}$, which would 319 leave no signal in the nuclear genome.

\section{Genomic footprints of a changing temperate forest}

322 We show that the story of the evolutionary diversification of modern Fagaceae can be 323 told through the lens of two unlinked genomes, each contributing unique inferences to 324 disentangle a complex combination of divergent and reticulate historical events that 325 unfolded through the Cenozoic. Further, historical migration events in temperate 326 lineages were revealed by discovery of three exceptions to the NW-OW plastome 327 pattern (Fig. 2). Chestnuts (Castanea) currently distributed across the Holarctic arose 328 in the OW and moved to the NW, while the Eurasian oak sublineages of sections 329 Quercus and Ponticae are NW in origin, consistent with RAD-seq analysis ${ }^{50}$. These 330 bidirectional land bridge crossings with unambiguous origins document the timing of 331 limited, but key dispersal events leading to the spread of modern Fagaceae forests 332 across the northern hemisphere ${ }^{101}$. While an untold number of extinctions will escape 333 this level of scrutiny, the reciprocal migrations of oak and chestnut species during the

334 Oligocene provide evidence for the origins of ecologically significant components of 335 northern hemisphere forests. The cascading ecological implications of biotic 336 exchanges of this magnitude at the organismal level await future study.

337 Hybridization, common throughout Fagales, may be adaptive at various stages 338 of diversification depending on patterns of persistent interfertility and range overlap 339 among lineages ${ }^{102}$. For Fagaceae, an early stage of widespread hybridization among 
340 ancestral elements of the HS clade is suggested by an enduring paleophylogeographic

341 signal in the plastomes of modern lineages. Soon after, a rapid burst of cladogenesis

342 at the base of the HS clade, tracked by the nuclear genome, generated the extant

343 lineages as resolved here. As reproductive isolation evolved across most of these

344 lineages, divergent evolution generated sets of exclusive plastome haplotypes within

345 the broader phylogeographic pattern observed here, except for the instances where

346 interfertile oak lineages experienced secondary contact during the Miocene. Within

347 several clades of Quercus, cytoplasmic-nuclear gene tree incongruities support

348 previous studies indicating an expanded role of hybridization in flowering plant 349 evolution $^{103-106}$.

350 We suggest that oaks and their chestnut relatives have been hybridizing for 351 millions of years. In Fagaceae, this is facilitated by small and evolutionarily stable 352 genomes, high levels of synteny, and a consistent chromosome number across taxa $353{ }^{107-112}$. In addition to conserved genomes and maintenance of some level of 354 interfertility, these lineages share other life-history traits with diverse and often 355 tropical tree genera that suggest the syngameon is functionally adaptive. Fagaceae 356 species in particular share evolutionary and ecological characteristics that may 357 promote adaptive introgression including generalized pollination systems, high levels 358 of fecundity, and widespread sympatry ${ }^{41,108,110}$.

359 Consequently, we document three main geographic areas of historical 360 introgression between oak sections as evidenced by plastome capture: western North 361 America, southeastern North America, and Eurasia. These areas are known to be 362 centers of phylogenetic diversity for the genus ${ }^{39}$ with extensive zones of sympatry and 363 evidence for convergent evolution of form in response to climate ${ }^{10,46}$. In two of these 364 areas, specifically Eurasia where the ranges of sections Ponticae and Quercus once 365 overlapped and the American southeast where sections Virentes and Quercus are still 366 known to hybridize, we present evidence from the nuclear genome that ancient 367 hybridization has left a signature of adaptive evolution. While more detailed study is 368 necessary to fully appreciate the impact these introgressed alleles may have had on 369 the modern oak landscape in these regions, ancient hybridization between the relictual 370 Q. pontica sublineage of sect. Ponticae and the widespread Eurasian sublineage of 371 sect. Quercus appears to have contributed to an increased diversification rate in 372 section Quercus during the Miocene (Fig 1; see also Hipp et al. ${ }^{39}$ ). This uptick in 373 speciation and the ecological opportunity available to the white oaks, a relative 
374 newcomer to the Old World, marks the rise and spread of a dominant deciduous

375 lineage bearing an introgressed nuclear genome into the forested ecosystems across

376 Eurasia.

378 Methods

379 Taxon sampling, DNA extraction and whole genome sequencing

380 We constructed a comprehensive Fagaceae dataset consisting of 122 individuals from

38191 species representing all eight currently recognized genera ${ }^{54,113}$. Complete taxon 382 sampling was achieved for three small genera: Chrysolepis (2 species), 383 Notholithocarpus (2 species) and Trigonobalanus (3 species). For the remaining 384 genera, representative samples for all major lineages were included: Fagus (2), 385 Castanea (5), Castanopsis (12) and Lithocarpus (10). For the well-studied genus 386 Quercus, extensive sampling (54 species) was conducted to represent all eight 387 recognized sections ${ }^{39,44}$. Several species were represented by multiple accessions 388 collected from different natural populations or cultivated plants. Betula pendula was 389 selected as an outgroup due to its close relationship to Fagaceae and the availability of 390 an assembled genome ${ }^{114}$. Accession information is provided in Supplementary Table 3911.

392 Total genomic DNA was extracted from silica-dried leaf tissue using BioTeke 393 Genomic DNA Extraction Kit (Beijing, China). High quality DNA was used to 394 constructed paired-end sequencing libraries with an insert size of 500-600 bp 395 according to the Illumina library preparation protocol. Sequencing was carried out on 396 the NovaSeq platform at Novogene (Beijing, China) to a coverage of $25-40 \times$ for all 397 samples. Short reads (150 bp pair-end) have been deposited to Genbank under 398 accession numbers XX-XX.

\section{Orthologous gene identification and nuclear alignment matrix assembly}

401 To obtain orthologous genes (OGs) for phylogenetic analysis, we performed a series 402 of critical search and filtering processes. There are four high quality genome 403 assemblies (chromosome-level) available in Fagaceae: Fagus sylvatica ${ }^{115}$, Castanea 404 mollissima ${ }^{116}$, Quercus robur ${ }^{91}$ and Quercus lobata ${ }^{117}$. These four assemblies together 405 with the outgroup species $B$. pendula ${ }^{114}$ were used to identify putative OGs in 406 OrthoFinder v2.3.12 $2^{118}$ with an E-value of $1 \mathrm{E}^{-5}$. Orthologous groups containing only 407 one sequence from each examined species were retained to minimize paralogs in 
408 subsequent phylogenetic analyses. Single copy genes (SCGs) identified by 409 OrthoFinder may still have duplicates, either as pseudogenes or un-annotated 410 functional genes in the genome. To identify and remove additional multiple-copy 411 genes, we blasted coding sequences (CDS) of SCGs against each of the five genomes 412 using BLAST $+{ }^{119}$. We filtered alignments using the following thresholds: E-value $<$ $4131 \mathrm{E}^{-5}$, alignment length $\geq 80 \%$ of the query sequence, and identity $\geq 80 \%$. We kept 414 CDS with only one hit in each of the five species. The retained CDS regions 415 identified as belonging to a single gene were concatenated for subsequent 416 phylogenetic analyses.

417 To generate the nuclear DNA sequences, we sequenced whole genomes of 117 418 individuals (Supplementary Table 1) to a coverage of $25-40 \times$ using the Illumina 419 NovaSeq platform and called genotypes in SCGs regions. We trimmed and filtered 420 raw reads using trimmomatic $\mathrm{v} 0.39^{120}$, mapped high quality reads to a reference 421 genome using BWA ${ }^{121}$, and called genotypes via HaplotypeCaller in GATK v4.2 ${ }^{122}$. 422 A simulation study found that the inclusion of nonpolymorphic positions in the 423 alignment and mapping short reads to multiple references could improve the accuracy 424 of phylogenetic inference ${ }^{123}$. Thus, we called all available sites (both variants and 425 invariants). To reduce the effects of reference bias, we used three reference genomes 426 for mapping and SNP calling in related species. The genome of $F$. sylvatica was used 427 as reference for genus Fagus, the Q. robur genome was used for genus Quercus, and 428 the Castanea mollissima genome was used for the remaining six genera. We only 429 considered sites with mapping quality $\geq 30$ and base quality $\geq 30$, and further filtered 430 variants using the following criteria: (1) homozygous genotypes with depth $<4$ or 431 heterozygous genotypes with depth $<20$ were assigned as missing; (2) sites with 432 mean depth $<5$ or $>100$ across all individuals were discarded; (3) sites with 433 proportion of heterozygous genotypes $>50 \%$ were excluded.

434 To obtain an aligned matrix of SCGs, we generated a 6-way whole genome 435 alignment based on the four reference genomes and two additional assemblies $(Q$. 436 lobata and $Q$. suber) following a lastZ/Multiz pipeline ${ }^{124,125}$. We used $Q$. robur as a 437 reference genome for genome alignment, and merged genotypes from mapping to 438 different references or extracted from different assemblies together according to their 439 relative positions on the $Q$. robur genome. The data matrix was then filtered by 440 excluding sites containing $\geq 10 \%$ missing data, and SCGs with length $<200 \mathrm{bp}$. 441 Alignments with divergent paralogous genes usually show elevated levels of 
442 polymorphism, thus we further excluded SCGs with polymorphism in the top $95^{\text {th }}$ 443 percentile (cutoff $=43.8 \%$ ). Every OG was presented in all sampled individuals with 444 no missing data. Our final dataset included 2124 SCGs with a total length of $4451,689,974 \mathrm{bp}$ for data analyses (Supplementary Table 2; Dryad Data Archive).

447 Evaluating the impacts of reference genomes on the accuracy of SNP calling and 448 phylogenetic reconstruction

449 We applied both empirical and simulation analyses to assess the impacts of the 450 reference genome on the accuracy of SNP calling and phylogenetic reconstruction. 451 The assembly of Castanea mollissima was used as the reference genome for SNP 452 calling in Castanea and the five genera (Chrysolepis, Castanopsis, Lithocarpus, 453 Notholithocarpus and Trigonobalanus) without available genome assemblies. To test 454 whether reference bias was introduced by using a divergent reference genome, we re455 called SNPs for these five genera by using $Q$. robur as reference, and compared 456 genotypes called from $Q$. robur with those from C. mollissima. Despite the slightly 457 higher rate of missing data $(9.29-9.72 \%)$ observed with using $Q$. robur as reference 458 genome compared to C. mollissima (3.96 - 4.27\%), 95.62 - 95.84\% genotypes were 459 identical between these two datasets (Supplementary Table 8). Identical tree 460 topologies also were generated based on the two datasets when using the same 461 phylogenetic method (data not shown), suggesting weak reference bias in our data.

462 To further monitor the accuracy of genotyping in the query dataset with 463 different divergence levels from the reference genome, we generated mutated 464 sequences (henceforth referred to as "mutated-sequence") by randomly adding $0.25 \%$ $465-20 \%$ mutations to the longest chromosome of $Q$. robur (chromosome 2, henceforth 466 referred to as "reference-sequence"). Next, we simulated 150bp pair-end reads from 467 each mutated-sequence with $30 \times$ coverage (close to our sequencing depth $25-40 \times$ ). 468 Simulated reads were mapped to the reference-sequence, and SNPs were called and 469 filtered using the same protocol as described above. For each simulated dataset, we 470 compared genotype calls to the mutated-sequence from which the datasets were 471 generated. To mimic the real data, SNPs called from the repetitive regions was 472 excluded from data analyses. The true positive (TP) rate was defined as TP/(TP+FP), 473 where TP is position identical to mutated-sequence, and FP (false positives) are called 474 genotypes different from mutated-sequence. The missing rate (MR) was defined as $475 \mathrm{MISS} / \mathrm{SIZE}$, where MISS is non-genotyped sites and SIZE is total sites $(\sim 51.2 \mathrm{Mb})$ in 
476 the reference-sequence after excluding masked repetitive regions. High TP rate ( $>$ $47797.7 \%)$ and low MR $(<1.5 \%)$ were found in datasets with divergence levels from 478 reference-sequence no more than 10\% (Supplementary Fig. 12). By extracting 479 sequences of the 2124 SCGs from the 6-way whole genome alignment, nucleotide 480 divergence was estimated as $7.46 \%-7.69 \%$ between most divergent genera (i.e. 481 Fagus vs. Quercus and Castanea) (Supplementary Table 9), genotypes called from 482 SCGs by using a divergent reference (e.g. using C. mollissima for other genera) 483 would not result in strong reference bias.

\section{Plastome assembly and alignment}

486 We assembled 117 plastomes during the course of this study and obtained five 487 additional plastomes from Genbank (Supplementary Table 1). Raw reads from whole 488 genome sequencing were used for de novo assembly of plastomes in NOVOPlasty $489 \mathrm{v} 4.2^{126}$. A ribulose-bisphosphate carboxylase $(r b c \mathrm{~L})$ gene sequence from Quercus 490 rubra was used as the seed sequence for assembly. Assembled plastomes were 491 annotated using the program PGA $^{127}$. The boundaries of inverted repeats and coding 492 regions of each annotated gene were determined in Geneious $7.1 .4^{128}$ by using the $Q$. 493 rubra plastome as a reference. Coding regions of 76 protein-coding genes present in 494 all species were extracted from the assemblies (Supplementary Table 10), aligned 495 using MAFFT 7.22129, and manually adjusted using Bioedit v.7.2 496 (https://bioedit.software.informer.com). Based on plant plastid genetic code, the 497 codon alignment was translated into amino acid sequences. A preliminary 498 phylogenetic analysis found two $Q$. ilex samples were placed as a sister group to all 499 other Fagaceae species except the genera Fagus and Trigonobalanus. This is likely an 500 artificial of clustering, because previous analyses with extensive sampling (26 501 individuals) spanning the geographic distribution of $Q$. ilex placed this species within 502 a clade formed by Eurasian oaks and genera Castanea and Castanopsis based on 503 plastid data ${ }^{53}$. Therefore, we excluded these two Q. ilex samples from subsequent 504 plastome analyses. Removing these two samples did not change the topology among 505 other species (data not shown). The plastome alignment is 65,814 bp in length, of 506 which 11,058 characters were polymorphic. A list of the 76 genes is presented in

507 Supplementary Table 10, the alignment of these genes can be found in Dryad508 archived data, and the plastome assemblies are deposited in Genbank under accession 509 numbers XX-XX. 
511 Phylogenetic analyses were conducted using Maximum Likelihood (ML) and

512 Bayesian approaches for concatenated nuclear and plastome data. Partitioned ML 513 analysis was performed using RAxML v8.2.12 $2^{123}$. The best-scoring ML tree was

514 found from 1000 ML trees, and topological robustness was evaluated by using 1000 515 non-parametric bootstrap replicates. Bayesian analysis was conducted in MrBayes 516 v3.2.6 $6^{130}$. Markov chain Monte Carlo (MCMC) runs were performed for 10 million 517 generations, and trees were sampled every 100 generations. The first 2,5000 (25\%) 518 trees were discarded as burn-in to ensure that the chains were stationary. The 519 remaining trees were used to generate a strict consensus tree and to calculate posterior 520 probabilities for each node.

521 PartitionFinder $2^{131}$ was used to determine the optimal partitioning strategy and 522 evolutionary model of each partition under the Akaike Information Criterion (AIC) ${ }^{132}$. 523 For nuclear DNA data, partitioning by gene yielded 35 partitions in the best scheme. 524 For plastome DNA data, full partitioning scheme by both locus and codon position 525 (each of the three codon positions in each gene as one partition) was examined, and 526 the best scheme contained 24 partitions. For plastome amino acid data, each gene was 527 considered as one partition, resulting in 12 partitions in the optimal scheme. In ML 528 analyses, the GTRGAMMA model was used for all DNA sequence partitions, and the 529 evolutionary models chosen by PartitonFinder2 were used for amino acid partitions. 530 For Bayesian analyses, the evolutionary model identified by PartitionFinder2 was 531 used for each DNA and amino acid partition. The models, partitions, and alignments 532 used for phylogenetic analysis can be found in Dryad Data Archive.

533 Two coalescent-based approaches were used to infer a species tree for 534 Fagaceae. First, we applied a summary statistic method using ASTRAL-III v5.7.3 ${ }^{133}$. 535 Gene trees were estimated from single-gene alignments using RAxML with 536 GTRGAMMA model and 1000 fast bootstrap replicates. Individual gene trees (best 537 trees) and bootstrap replicates were used to estimate a species tree in ASTRAL-III 538 with 1000 coalescent bootstrap replicates. Following Zhang et al. ${ }^{133}$, branches with 539 low support were removed to improve the accuracy of tree inference. We tested 540 different thresholds by collapsing branches with support less than $10 \%, 20 \%, 30 \%$, $54140 \%$ and 50\%, and obtained near-identical tree topologies (data not shown). The tree 542 generated by ASTRAL-III with 50\% threshold is presented. 
543 SVDquartets ${ }^{134}$, a method based on site pattern frequencies and algebraic 544 statistics implemented in PAUP v4.0a152 ${ }^{135}$ was additionally used to estimate a 545 species tree. This method was originally designed for SNP data, but also performed 546 well on large multiple-locus datasets ${ }^{134}$. The concatenated nuclear data matrix was 547 used as input for SVDquartets. All possible quartets were evaluated, and clade 548 support was assessed using 500 bootstrap replicates.

\section{Divergence time and diversification rate estimation}

551 Divergence time estimation was conducted for both plastome and nuclear datasets 552 using MCMCTree v4.9j in the PAML package ${ }^{136}$. MCMCTree estimates divergence 553 times using an approximate likelihood method, and is computationally efficient with 554 large genomic data ${ }^{137}$. The MCMC chains were first run for 3 million generations as 555 burn-in, and then were sampled every 400 generations until a total of 25,000 samples 556 were collected (10 million generations). Tracer and LogCombiner were used to 557 confirm the convergence across each run and ensure the ESS of all parameters were 558 greater than 200. For each of the plastome and nuclear datasets, three independent 559 runs with different seeds were compared for convergence, and similar results were 560 generated.

561 For nuclear DNA data, we divided the 2124 nuclear genes into three partitions 562 according to substitution rates estimated by Baseml (package in PAML) with a strict 563 molecular clock and then applied an uncorrelated rate model (clock $=2$ in 564 MCMCTree) to infer divergence times. We used priors of $G(1,6.1677)$ for the 565 overall substitution rates (rgene_gamma), $G(2,5,1)$ for the rate-drift parameter 566 (sigma2_gamma). Because concatenated and coalescent analyses revealed different 567 relationships among genera Quercus + Notholithocarpus, Lithocarpus and Chrysolepis, 568 we constrained each alternative topology and constructed the ML reference tree for 569 dating. For plastome data, we treated all $76 \mathrm{cp}$ genes as one partition, and estimated 570 divergence times by using the ML tree as reference under an uncorrelated rate model.

571 We set priors of rgene_gamma and sigma2_gamma parameters as $\mathrm{G}(1,41.667)$ and $572 \mathrm{G}(2,5,1)$, respectively.

573 Based on results of Xiang et al. ${ }^{64}$, the root age of Fagaceae was constrained to 57495.5 - 101.2 MYA for both plastome and nuclear data. For nuclear data, we further 575 added six additional widely accepted fossil calibrations (Supplementary Table 3). For 576 the plastome analysis, only two calibrations could be used due to non-monophyletic 
577 lineages in the plastome tree (Supplementary Table 3). For species with multiple 578 samples, we chose one individual for dating the nuclear DNA tree, while retaining all 579 individuals for dating the plastome tree because many species were not monomorphic 580 for their plastome.

581 To estimate the diversification rate of Fagaceae, we applied Bayesian Analysis 582 of Macroevolutionary Mixture (BAMM) ${ }^{138}$. The time tree estimated by MCMCtree 583 was used as an input tree. To account for incomplete taxon sampling, we calculated 584 sampling fraction of each genus and each section of genus Quercus based on the 585 number of species recorded in previous reports ${ }^{39,113}$, and then added un-sampled taxa 586 to a random position in each corresponding lineage (Supplementary Table 11). The 587 BAMM analyses were run for 10 million generations, saving every 1000 generations.

588 The first $30 \%$ samples were discarded as burn-in, and the remaining samples were 589 summarized and plotted using BAMMtools ${ }^{138}$.

\section{Topological concordance analyses}

592 To evaluate the conflicts between nuclear gene trees and the species tree, we first 593 calculated the internode certainty all (ICA) to quantify the degree of conflict on each 594 node between a target tree and gene trees ${ }^{139}$. ICA values close to 1 indicate strong 595 concordance for the bipartition defined by a given internode, while ICA values close 596 to 0 indicate strong conflict. Negative ICA values indicate that the defined bipartition 597 conflict with other high frequent bipartitions. The ICA values were estimated in 598 RAxML and the species tree found by ASTRAL-III was used as the target tree. We 599 further summarized the number of conflicting and concordant bipartitions with 600 PHYPARTS ${ }^{140}$, using the species tree estimated by ASTRAL-III and the individual 601 gene trees.

603 Evaluation of substitutional saturation and codon-usage bias within the chloroplast 604 dataset

605 To investigate whether base substitution saturation biased the accuracy of 606 phylogenetic inference in plastome phylogenetic analyses, we estimated the amount 607 of substitution saturation using methods detailed in Xia et al. ${ }^{141}$. This involved 608 employing critical index of substitution saturation (ISSc) that defines a threshold for 609 significant saturation in the data. From the data of 76 chloroplast genes, we assessed 610 the level of substitution saturation for codon 12 and codon 3 using the program 
611 DAMBE7 $7^{142}$, and found that there was sufficient phylogenetic information at all 612 codon positions (Supplementary Table 12).

613 To investigate how synonymous codon usage varies among Fagaceae species, 614 and whether synonymous codon biases have resulted in artificial and random 615 phylogenetic inference, we measured Relative Synonymous Codon Usage (RSCU) 616 values using GCUA ${ }^{143}$. RSCU is defined as the ratio of the observed codon 617 appearance to the number expected given that all synonymous codons appear with 618 uniform frequency. We found similar level of GC content and variation in codon bias 619 across Fagaceae species (Supplementary Fig. 13). These results suggested that 620 Fagaceae plastid genomes are highly conserved, and the plastid-based analyses would 621 be not biased due to substitution saturation or compositional heterogeneity among 622 species.

\section{Coalescent simulation}

625 To test whether incomplete lineage sorting (ILS) alone could explain the 626 incongruence between plastome tree and nuclear species tree, we followed Folk et $627 \mathrm{al}^{144}$ to simulate 10,000 plastome trees under the coalescent model using 628 DENDROPY v.4.1.0 $0^{145}$. The ASTRAL-III tree was used as a guide tree for the 629 simulation. To simulate plastome trees, branch lengths were scaled by a factor of four 630 to account for the haploidy and maternal inheritance of the plastome. Clade 631 frequencies of simulated trees were summarized using PHYPARTS ${ }^{140}$. In the scenario 632 of ILS alone, the topology from our empirical plastome tree should be present in 633 simulated trees with high frequency; if gene flow is present, the topology recovered in 634 our empirical tree should be absent or at very low frequency in the simulated trees. 635 Following previous studies ${ }^{96,146}$, we also counted the number of extra lineages in 636 observed and simulated trees using the function deep-coal_count in Phylonet v2.4 ${ }^{147}$. 637 In the case that gene flow is present, more extra lineages are expected in the observed 638 trees relative to simulated trees.

\section{Gene flow analyses}

641 To detect potential gene flow between species, we performed ABBA-BABA statistic 642 tests in Dsuite ${ }^{148}$. These analyses take advantage of a four taxon statement ((H1, $643 \mathrm{H} 2) \mathrm{H} 3) \mathrm{H} 4)$. With $\mathrm{H} 4$ as the outgroup, $\mathrm{H} 1$ and $\mathrm{H} 2$ are treated as a pair of sister species 644 and $\mathrm{H} 3$ is tested as the species with potential gene flow with $\mathrm{H} 1$ or $\mathrm{H} 2$. The number 
645 of sites with allele patterns of ABBA and BABA are tallied. The $D$ statistic is derived 646 from calculating $D=(\mathrm{nABBA}-\mathrm{nBABA}) /(\mathrm{nABBA}+\mathrm{nBABA})$, where nABBA and $647 \mathrm{nBABA}$ are the total number of sites with patterns of ABBA and BABA, 648 respectively ${ }^{149,150}$. A negative $D$ value indicates gene flow between $\mathrm{H} 1$ and $\mathrm{H} 3$, a 649 positive $D$ value indicates gene flow between $\mathrm{H} 2$ and $\mathrm{H} 3$, and $D=0$ indicates no gene 650 flow $^{149,150}$. Because ABBA-BABA test assumes a sister relationship between $\mathrm{H} 1$ and $651 \mathrm{H} 2$, we restricted our analyses by sampling H1 and H2 from same genera, or same 652 sections within genus Quercus. In addition, because H1 and H2 are sister species, the 653 sites with the pattern of BBAA are expected to be larger than ABBA and BABA 654 patterns. We further filtered trios that violated this assumption, and applied ABBA655 BABA test to 25882 trios extracted from the species tree. To account for multiple 656 testing, we corrected $P$-values with Benjamini-Hochberg FDR ${ }^{151}$. For a pair of species 657 involved in multiple tested trios (for example, while $\mathrm{H} 2$ and $\mathrm{H} 3$ are fixed, there may 658 be different $\mathrm{H} 1$ taxa available, thus different $D$ values for $\mathrm{H} 2$ and $\mathrm{H} 3$ may be 659 generated), the estimated $D$ value with lowest FDR was retained. An individual of 660 Trigonobalanus doichangensis was used as an outgroup for all tests. To test how 661 outgroup choice influenced the analysis, we also used an individual of 662 Notholithocapus densiflorus in tests within Quercus and obtained results similar to 663 those using T. doichangensis (data not shown).

664 To further explore the reticulate evolutionary histories within Fagaceae, we 665 inferred species networks using $\mathrm{SNaQ}^{152}$ implemented in the package 666 PhyloNetworks ${ }^{153}$. SNaQ is a pseudolikelihood method, which estimates a 667 phylogenetic network while accounting for both ILS and gene flow ${ }^{152}$. We reduced 668 the dataset to a computationally tractable size ${ }^{154}$, and generated four sub-datasets each 669 with 15-17 taxa sampled. For each sub-dataset, we sampled species showing 670 inconsistent placement between nuclear and plastome trees. The first one focused on 671 relationships within subgenus Quercus and a sample of 16 species (Supplementary 672 Fig. 10). The second one focused on the relationship within subgenus Cerris and a 673 sample of 15 species (Supplementary Fig. 10). The third one focused on the 674 relationships among genera Castanea, Castanopsis, Lithocarpus, and Quercus, and 675 the forth one other focused on the relationship among genera Chrysolepis, 676 Notholithocarpus, and Quercus (Supplementary Fig. 10). One individual gene trees 677 generated by RAxML were used as input, and nested analyses were performed 678 allowing for zero $(h=0)$ to four $(h=4)$ hybridization events. Each nested analysis 
679 was optimized by 10 independent runs, and the best fitting model was selected based 680 on the log pseudolikelihood score.

681 To investigate the genomic pattern of introgressed loci, we quantified the 682 distribution of phylogenetic signal for conflicting topologies across nuclear gene 683 trees, and then mapped loci supporting alternative partitions to the $Q$. robur genome ${ }^{91}$. 684 Following Shen et al. ${ }^{155}$, we calculated site-wise log-likelihood scores for the primary 685 and alternative topologies in our concatenated matrix using the "-f G" command in 686 RAxML. After that, the difference in site-wise log-likelihood scores ( $\triangle \mathrm{SLS}$ ) between 687 topologies were summed across sites in each gene, generating gene-wise log688 likelihood scores ( $\triangle$ GLS). For each node of interest, the primary topology was 689 defined as the species tree recovered by ASTRAL-III, and the alternative topologies 690 were ML trees constrained to recover the most common conflicting bipartitions.

\section{Identity-by-descent (IBD) analyses}

693 We performed IBD analyses based on genome-wide SNP data in the genus Quercus.

694 By using a same SNP calling and filtering procedure described above (see section 695 "Orthologous gene identification and nuclear alignment matrix assembly"). Raw 696 reads of Quercus species were trimmed using Trimmomatic v0.38 ${ }^{120}$, aligned to $Q$. 697 robur reference genome assembly ${ }^{91}$ using BWA $^{121}$, and called genotypes using 698 GATK v4.1 ${ }^{156}$. We applied a strict filtering process to remove low quality SNPs. We 699 removed all sites located in repetitive regions of the $Q$. robur reference genome ${ }^{91}$, and 700 discarded all indels and multiallelic SNPs. We further set genotypes supported by less 701 than four reads as missing data, and deleted SNPs with mean depth $<5$ or $>100$, or 702 genotyped in less than half of individuals, or proportion of called heterozygous 703 genotypes $>50 \%$. Finally, we obtained 34,250,467 high quality SNPs for IBD 704 analyses.

705 We used Beagle v4.1 $1^{157}$ to phase and impute the SNP data, and uncover shared 706 IBD blocks between species. The following parameters were used for IBD analyses in 707 Beagle: window $=100,000$; overlap $=10,000$; ibdtrim $=100$; ibdlod $=5$. To compare 708 the recombination rate between IBD blocks and genomic background, we used a 709 genetic map of $Q$. robur developed by Plomion et al. ${ }^{91}$. We smoothed the 710 recombination rate across the genome to $200 \mathrm{~kb}$, and then mapped IBD blocks to the 711 genetic map. For each IBD block, we obtained the recombination rate on middle 
712 points of the block, and then used this value as the recombination rate for the whole 713 IBD block.

714 To test whether the IBDs shared between sections are under selection, we 715 calculated the probability of a selectively neutral haplotype with a given length shared 716 by two sections after introgression. If the IBD blocks were significant longer than the 717 neutral haplotype, they were most likely maintained by selection after introgression. 718 Following Huerta-Sanchez et al. ${ }^{86}$, the probability for each shared IBD block was 719 estimated as: 1 - GammaCDF $(L$, shape $=2$, rate $=$ lambda), where the GAmmaCDF is 720 the Gamma distribution function and arguments are given in parentheses. The rate 721 parameter lambda was estimated as: lambda $=r *(T / G)$, where $r$ is recombination 722 rate, $T$ is the time of gene flow occurred, and $\mathrm{G}$ is the generation time. To calculate 723 the time of gene flow introduced shared IBDs between oak sections, we calculated the 724 genetic divergence $\left(d_{\mathrm{XY}}\right)$ between sections (i.e. $Q$. pontica vs. European and Asian 725 white oaks, North American white oaks vs. section Virentes and Q. sadleriana) on 726 shared IBD blocks. The estimated mean values of $d_{\mathrm{XY}}$ was $0.011-0.018$, which was 727 transformed to $2.8-4.5$ millions years based on a mutation rate of $2 \times 10^{-9}$ per site per 728 year ${ }^{10}$. Thus, we roughly used 3 million years for the time of introgression. Using a 729 recombination rate of $1 \times 10^{-8}$ estimated from $Q$. robur genetic map (total length of 730 genetic map is $740 \mathrm{cM}$, and the genome size is $804 \mathrm{Mb})^{91}$, and assuming a generation 731 time of 50 years, we get lambda $=1 \times 10^{-8} \times\left(3 \times 10^{6} / 50\right)=6 \times 10^{-4}$. We calculated

732 the probability for each IBD blocks and corrected multiple testing using Benjamini733 Hochberg FDR ${ }^{151}$.

734 To examine whether functional classes of genes were overrepresented in IBD 735 blocks under selection, we performed GO analyses using the R package topGO 2.43.0 736 (http://www.bioconductor.org/). We applied Fisher's exact test to estimate the 737 statistical significance of enrichment, and corrected multiple testing by Benjamini738 Hochberg FDR ${ }^{151}$. A cutoff of FDR $<0.01$ was used to determine the significance of 739 GO enrichment.

\section{References}

7421 Sun, J. et al. Synchronous turnover of flora, fauna, and climate at the Eocene743 Oligocene Boundary in Asia. Scientific Reports 4, 7463 (2014). 
7442 Tiffney, B. H. Perspectives on the origin of the floristic similarity be tween 745 eastern Asia and eastern North America. Journal of the Arnold Arboretum 66, $746 \quad$ 73-94 (1985).

7473 Tiffney, B. H. The Eocene North Atlantic land bridge: its importance in 748 Tertiary and modern phytogeography of the Northern Hemisphere. Journal of 749 the Arnold Arboretum 66, 243-273 (1985).

7504 Donoghue, M. J. A phylogenetic perspective on the distribution of plant 751 diversity. Proceedings of the National Academy of Sciences of the United $752 \quad$ States of America 105, 11549-11555 (2008).

7535 Edwards, E. J. et al. Convergence, consilience, and the evolution of temperate 754 deciduous forests. American Naturalist 190, S87-S104 (2017).

7556 Segovia, R. A. et al. Freezing and water availability structure the evolutionary 756 diversity of trees across the Americas. Science Advances 6, eaaz5373 (2020).

7577 Tiffney, B. H. \& Manchester, S. R. The use of geological and paleontological 758 evidence in evaluating plant phylogeographichypotheses in the Northern 759 Hemisphere Tertiary. International Journal of Plant Sciences 162, S3-S17 $760 \quad$ (2001).

7618 Axelrod, D. I. Biogeography of oaks in the Arcto-Tertiary province. Annals of 762 the Missouri Botanical Garden 70, 629-657 (1983).

7639 Axelrod, D. I., Ai-Shehbaz, I. \& Raven, P. H. History of the modern flora of $764 \quad$ China. (Springer, 1996).

76510 Cavender-Bares, J. Diversification, adaptation, and community assembly of 766 the American oaks (Quercus), a model clade for integrating ecology and 767 evolution. New Phytologist 221, 669-692 (2019).

76811 Delcourt, H. R. \& A., D. P. North American terrestrial vegetation. 769 (Cambridge University Press, 2000).

77012 Olson, J. S., Watts, J. A. \& Allison, L. J. Carbon in live vegetation of major $771 \quad$ world ecosystems. (1983).

77213 Soepadmo, E. Flora Malesiana Series I. Vol. 7 (Noordhoff International 773 Publishing, 1972).

77414 Vogt, K. A. et al. Review of root dynamics in forest ecosystems grouped by 775 climate, climatic forest type and species. Plant and Soil 187, 159-219 (1996).

77615 Whitmore, T. C. Tropical rain forest of the Far East. (Oxford University $777 \quad$ Press, 1984). 
16 Zhu, H. Ecological and biogeographical studies on the tropical rain forest of south Yunnan, SW China with a special reference to its relation with rain forests of tropical Asia. Journal of Biogeography 24, 647-662 (1997). Averill, C., Bhatnagar, J. M., Dietze, M. C., Pearse, W. D. \& Kivlin, S. N. Global imprint of mycorrhizal fungi on whole-plant nutrient economics. Proceedings of the National Academy of Sciences of the United States of America 116, 23163-23168 (2019).

18 Martin, F., Kohler, A., Murat, C., Veneault-Fourrey, C. \& Hibbett, D. S. Unearthing the roots of ectomycorrhizal symbioses. Nature Reviews Microbiology 14, 760-773 (2016).

19 Smith, S. E. \& Read, D. J. Mycorrhizal Symbiosis, 2nd edn. (Academic Press, 1997).

20 Abrahamson, W. G. \& Melika, G. Gall-inducing insects (Cynipinae) provide insights into plant systematic relationships. American Journal of Botany 85, 111-111 (1998).

Raman, A. Nutritional diversity in gall-inducing insects and their evolutionary relationships with flowering plants. International Journal of Ecology and Environmental Sciences 22, 133-143 (1996). Stone, G. N. et al. Extreme host plant conservatism during at least 20 million years of host plant pursuit by oak gallwasps. Evolution 63, 854-869 (2009). Johnson, W. C. \& Webb, T. The role of bluejays (Cyanocitta cristata L.) in the postglacial dispersal of fagaceous trees in eastern North America. Journal of Biogeography 16, 561-571 (1989).

Koenig, W. D. \& Haydock, J. Oaks, acorns, and the geographical ecology of acorn woodpeckers. Journal of Biogeography 26, 159-165 (1999).

25 Payne, J. \& Francis, C. M. A Field Guide to the Mammals of Borneo. (Sabah Society with World Wildlife Fund Malaysia, 1985).

6 Steele, M. A. Oak Seed Dispersal. (The Johns Hopkins University Press, 2021).

7 Vander Wall, S. B. The evolutionary ecology of nut dispersal. Botanical Review 67, 74-117 (2001). seed-dispersing animals. Philosophical Transactions of the Royal Society BBiological Sciences 365, 989-997 (2010). 
81229 Barrón, E. et al. in Oaks Physiological Ecology. Exploring the Functional 813 Diversity of Genus Quercus L. (eds Eustaquio Gil-Pelegrín, José Javier 814 Peguero-Pina, \& Domingo Sancho-Knapik) 39-105 (Springer International 815 Publishing, 2017).

81630 Crepet, W. L. \& Nixon, K. C. Earliest megafossil evidence of Fagaceae: 817 phylogenetic and biogeographic implications. American Journal of Botany 76, 818 842-855 (1989).

81931 Denk, T. \& Grimm, G. W. Significance of pollen characteristics for 820 infrageneric classification and phylogeny in Quercus (Fagaceae). 821 International Journal of Plant Sciences 170, 926-940 (2009).

82232 Denk, T., Grímsson, F. \& Zetter, R. Fagaceae from the early Oligocene of 823 Central Europe: Persisting new world and emerging old world biogeographic 824 links. Review of Palaeobotany and Palynology 169, 7-20 (2012).

82533 Grímsson, F., Grimm, G. W., Zetter, R. \& Denk, T. Cretaceous and Paleogene 826 Fagaceae from North America and Greenland: Evidence for a Late Cretaceous 827 split between Fagus and the remaining Fagaceae. Acta Palaeobotanica 56, 828 247-305 (2016).

82934 Jones, J. H. Evolution of the Fagaceae: the implications of foliar features. $830 \quad$ Annals of the Missouri Botanical Garden 73, 228-275 (1986).

83135 Manchester, S. R. Biogeographical relationships of North American Tertiary 832 floras. Annals of the Missouri Botanical Garden 86, 472-522 (1999).

83336 Sadowski, E. M., Schmidt, A. R. \& Denk, T. Staminate inflorescences with in 834 situ pollen from Eocene Baltic amber reveal high diversity in Fagaceae (oak 835 family). Willdenowia 50, 405-517 (2020).

83637 Bouchal, J., Zetter, R., Grimsson, F. \& Denk, T. Evolutionary trends and 837 ecological differentiation in early Cenozoic Fagaceae of western North 838 America. American Journal of Botany 101, 1332-1349 (2014).

83938 Gandolfo, M. A., Nixon, K. C., Crepet, W. L. \& Grimaldi, D. A. A late 840 Cretaceous fagalean inflorescence preserved in amber from New Jersey. 841 American Journal of Botany 105, 1424-1435 (2018).

84239 Hipp, A. L. et al. Genomic landscape of the global oak phylogeny. New $843 \quad$ Phytologist 226, 1198-1212 (2020). 
84440 Wilf, P., Nixon, K. C., Gandolfo, M. A. \& Cuneo, N. R. Eocene Fagaceae 845 from Patagonia and Gondwanan legacy in Asian rainforests. Science 364, 846 eaaw5139 (2019).

84741 Petit, R. J. \& Hampe, A. Some evolutionary consequences of being a tree. $848 \quad$ Annual Review of Ecology Evolution and Systematics 37, 187-214 (2006).

84942 Smith, S. A. \& Donoghue, M. J. Rates of molecular evolution are linked to life 850 history in flowering plants. Science 322, 86-89 (2008).

85143 Denk, T. \& Grimm, G. W. The biogeographic history of beech trees. Review 852

44 Denk, T., Grimm, G. W., Manos, P. S., Deng, M. \& Hipp, A. L. in Oaks 854 Physiological Ecology. Exploring the Functional Diversity of Genus Quercus L. Vol. 7 Tree Physiology (eds E. GilPelegrin, J. J. PegueroPina, \& D. SanchoKnapik) 13-38 (Springer International Publishing, 2017). Deng, M., Jiang, X. L., Hipp, A. L., Manos, P. S. \& Hahn, M. Phylogeny and biogeography of East Asian evergreen oaks (Quercus section Cyclobalanopsis; Fagaceae): Insights into the Cenozoic history of evergreen broad-leaved forests in subtropical Asia. Molecular Phylogenetics and Evolution 119, 170-181 (2018).

6 Hipp, A. L. et al. Sympatric parallel diversification of major oak clades in the

86848 Hauser, D. A., Keuter, A., McVay, J. D., Hipp, A. L. \& Manos, P. S. The Americas and the origins of Mexican species diversity. New Phytologist 217, 439-452 (2018).

Crowl, A. A. et al. Uncovering the genomic signature of ancient introgression between white oak lineages (Quercus). New Phytologist 226, 1158-1170 (2020).

evolution and diversification of the red oaks of the California Floristic Province (Quercus section Lobatae, series Agrifoliae). American Journal of Botany 104, 1581-1595 (2017).

49 McVay, J. D., Hauser, D., Hipp, A. L. \& Manos, P. S. Phylogenomics reveals a complex evolutionary history of lobed-leaf white oaks in western North America. Genome 60, 733-742 (2017).

50 McVay, J. D., Hipp, A. L. \& Manos, P. S. A genetic legacy of introgression confounds phylogeny and biogeography in oaks. Proceedings of the Royal Society B 284, 20170300 (2017). 
87851 Manos, P. S., Doyle, J. J. \& Nixon, K. C. Phylogeny, biogeography, and 879 processes of molecular differentiation in Quercus subgenus Quercus 880 (Fagaceae). Molecular Phylogenetics and Evolution 12, 333-349 (1999).

88152 Pham, K. K., Hipp, A. L., Manos, P. S. \& Cronn, R. C. A time and a place for 882 everything: Phylogenetic history and geography as joint predictors of oak 883 plastome phylogeny. Genome 60, 720-732 (2017).

88453 Simeone, M. C. et al. Plastome data reveal multiple geographic origins of 885 Quercus Group Ilex. PeerJ 4, e1897 (2016).

88654 Oh, S. H. \& Manos, P. S. Molecular phylogenetics and cupule evolution in $887 \quad$ Fagaceae as inferred from nuclear CRABS CLAW sequences. Taxon 57, 434$888451(2008)$.

88955 Renne, P. R. et al. Time scales of critical events around the Cretaceous$890 \quad$ Paleogene boundary. Science 339, 684-687 (2013).

89156 Koenen, E. J. M. et al. The origin of the Legumes is a complex paleopolyploid 892 phylogenomic tangle closely associated with the Cretaceous-Paleogene (K893 Pg) mass extinction event. Systematic Biology 70, 508-526 (2021).

89457 Wang, W. et al. Menispermaceae and the diversification of tropical rainforests 895 near the Cretaceous-Paleogene boundary. New Phytologist 195, 470-478 896 (2012).

89758 Suh, A., Smeds, L. \& Ellegren, H. The dynamics of incomplete lineage sorting 898 across the ancient adaptive radiation of Neoavian birds. PLoS Biology 13, 899 e1002224 (2015).

90059 Feng, Y. J. et al. Phylogenomics reveals rapid, simultaneous diversification of 901 three major clades of Gondwanan frogs at the Cretaceous-Paleogene 902 boundary. Proceedings of the National Academy of Sciences of the United $903 \quad$ States of America 114, E5864-E5870 (2017).

90460 Alfaro, M. E. et al. Explosive diversification of marine fishes at the 905 Cretaceous-Palaeogene boundary. Nature Ecology \& Evolution 2, 688-696 906 (2018).

90761 Meredith, R. W. et al. Impacts of the Cretaceous terrestrial revolution and 908 KPg extinction on mammal diversification. Science 334, 521-524 (2011).

90962 Martinez, I. \& Gonzalez-Taboada, F. Seed dispersal patterns in a temperate 910 forest during a mast event: performance of alternative dispersal kernels. $911 \quad$ Oecologia 159, 389-400 (2009). 
91263 Larson-Johnson, K. Phylogenetic investigation of the complex evolutionary 913 history of dispersal mode and diversification rates across living and fossil $914 \quad$ Fagales. New Phytologist 209, 418-435 (2016).

91564 Xiang, X. G. et al. Large-scale phylogenetic analyses reveal fagalean 916 diversification promoted by the interplay of diaspores and environments in the 917 Paleogene. Perspectives in Plant Ecology, Evolution and Systematics 16, 101$918 \quad 110(2014)$.

91965 Casanovas-Vilar, I. et al. Oldest skeleton of a fossil flying squirrel casts new $920 \quad$ light on the phylogeny of the group. Elife 7, e39270 (2018).

92166 Huchon, D. et al. Rodent phylogeny and a timescale for the evolution of 922 glires: Evidence from an extensive taxon sampling using three nuclear genes. 923 Molecular Biology and Evolution 19, 1053-1065 (2002).

92467 Upham, N. S., Esselstyn, J. A. \& Jetz, W. Inferring the mammal tree: Species925 level sets of phylogenies for questions in ecology, evolution, and conservation. $926 \quad$ PLoS Biology 17, e3000494 (2019).

92768 Jonsson, K. A. et al. A supermatrix phylogeny of corvoid passerine birds 928 (Ayes: Corvides). Molecular Phylogenetics and Evolution 94, 87-94 (2016).

92969 Prum, R. O. et al. A comprehensive phylogeny of birds (Aves) using targeted 930 next-generation DNA sequencing. Nature 526, 569-573 (2015).

93170 Benz, B. W., Robbins, M. B. \& Peterson, A. T. Evolutionary history of 932 woodpeckers and allies (Aves : Picidae): Placing key taxa on the phylogenetic 933 tree. Molecular Phylogenetics and Evolution 40, 389-399 (2006).

93471 Lutzoni, F. et al. Contemporaneous radiations of fungi and plants linked to 935 symbiosis. Nature Communications 9, 5451 (2018).

93672 Bonfante, P. \& Genre, A. Mechanisms underlying beneficial plant-fungus 937 interactions in mycorrhizal symbiosis. Nature Communications 1, 48 (2010).

93873 Miyauchi, S. et al. Large-scale genome sequencing of mycorrhizal fungi 939 provides insights into the early evolution of symbiotic traits. Nature $940 \quad$ Communications 11, 5125 (2020).

94174 Varga, T. et al. Megaphylogeny resolves global patterns of mushroom 942 evolution. Nature Ecology \& Evolution 3, 668-678 (2019).

94375 Yang, Y. Y., Qu, X. J., Zhang, R., Stull, G. W. \& Yi, T. S. Plastid 944 phylogenomic analyses of Fagales reveal signatures of conflict and ancient 

(2021).

94776 Whitmore, L. T. E. \& Schaal, B. A. Interspecific gene flow in sympatric oaks.

948 Proceedings of the National Academy of Sciences of the United States of 949 America 88, 2540-2544 (1991).

95077 Kremer, A. \& Hipp, A. L. Oaks: an evolutionary success story. New 951 Phytologist 226, 987-1011 (2020).

95278 Petit, R. et al. Chloroplast DNA variation in European white oaks 953 phylogeography and patterns of diversity based on data from over 2600 954 populations. Forest Ecology and Management 176, 595-599 (2003).

95579 Petit, R. et al. Chloroplast DNA footprints of postglacial recolonization by 956 oaks. Proceedings of the National Academy of Sciences of the United States of 957 America 94, 9996-10001 (1997).

95880 Petit, R. J. \& Excoffier, L. Gene flow and species delimitation. Trends in $959 \quad$ Ecology \& Evolution 24, 386-393 (2009).

96081 Premoli, A. C., Mathiasen, P., Cristina Acosta, M. \& Ramos, V. A. 961 Phylogeographically concordant chloroplast DNA divergence in sympatric 962 Nothofagus s.s. How deep can it be? New Phytologist 193, 261-275 (2012).

96382 Tsuda, Y., Semerikov, V., Sebastiani, F., Vendramin, G. G. \& Lascoux, M. 964 Multispecies genetic structure and hybridization in the Betula genus across 965 Eurasia. Molecular Ecology 26, 589-605 (2017).

96683 Zhang, B. W. et al. Phylogenomics reveals an ancient hybrid origin of the 967 persian walnut. Molecular Biology and Evolution 36, 2451-2461 (2019).

96884 Bock, R. Witnessing genome evolution: Experimental reconstruction of 969 endosymbiotic and horizontal gene transfer. Annual Review of Genetics 51, 1$97022(2017)$.

97185 Hill, W. G. Disequilibrium among several linked neutral genes in finite 972 population. II. Variances and covariances of disequilibria. Theoretical 973 Population Biology 6, 184-198 (1974).

97486 Huerta-Sanchez, E. et al. Altitude adaptation in Tibetans caused by 975 introgression of Denisovan-like DNA. Nature 512, 194-197 (2014).

$97687 \quad$ Huang, Y. et al. Megabase-scale presence-absence variation with Tripsacum 977 origin was under selection during maize domestication and adaptation. $978 \quad$ Genome Biology 22, 237 (2021). 
97988 Zhang, X. et al. The history and evolution of the Denisovan-EPAS1 haplotype 980 in Tibetans. Proceedings of the National Academy of Sciences of the United States of America 118, e2020803118 (2021).

98289 Cavender-Bares, J., Gonzalez-Rodriguez, A., Pahlich, A., Koehler, K. \& 983 Deacon, N. Phylogeography and climatic niche evolution in live oaks (Quercus series Virentes) from the tropics to the temperate zone. Journal of Biogeography 38, 962-981 (2011).

98690 Chen, D. et al. Phylogeography of Quercus variabilis based on chloroplast dna sequence in East Asia: Multiple glacial refugia and mainland-migrated island populations. PLoS One 7, e47268 (2012).

98991 Plomion, C. et al. Oak genome reveals facets of long lifespan. Nature Plants 4, 440-452 (2018).

92 Leroy, T. et al. Adaptive introgression as a driver of local adaptation to climate in European white oaks. New Phytologist 226, 1171-1182 (2020).

99393 Maxwell, L. M., Walsh, J., Olsen, B. J. \& Kovach, A. I. Patterns of 994 introgression vary within an avian hybrid zone. BMC Ecology and Evolution 21, $14(2021)$.

99694 Hewitt, G. M. Hybrid zones - natural laboratories for evolutionary studies. Trends in Ecology \& Evolution 3, 158-167 (1988).

99895 Gernandt, D. S., Resendiz Arias, C., Terrazas, T., Aguirre Dugua, X. \& 999 Willyard, A. Incorporating fossils into the Pinaceae tree of life. American Journal of Botany 105, 1329-1344 (2018).

Rose, J. P., Toledo, C. A. P., Lemmon, E. M., Lemmon, A. R. \& Sytsma, K. J. Out of sight, out of mind: Widespread nuclear and plastid-nuclear discordance in the flowering plant genus Polemonium (Polemoniaceae) suggests

101199 Sork, V. L. et al. Phylogeny and introgression of California scrub white oaks (Quercus section Quercus). International Oaks Journal 27, 61-74 (2016). 
1013100 Quang, N. D., Ikeda, S. \& Harada, K. Nucleotide variation in Quercus $1014 \quad$ crispula Blume. Heredity 101, 166-174 (2008).

1015101 Graham, A. The role of land bridges, ancient environments, and migrations in 1016 the assembly of the North American flora. Journal of Systematics and 1017 Evolution 56, 405-429 (2018).

1018102 Suarez-Gonzalez, A., Lexer, C. \& Cronk, Q. C. B. Adaptive introgression: A 1019 plant perspective. Biology Letters 14 (2018).

1020103 Abbott, R. J., Barton, N. H. \& Good, J. M. Genomics of hybridization and its 1021 evolutionary consequences. Molecular Ecology 25, 2325-2332 (2016).

1022104 Goulet, B. E., Roda, F. \& Hopkins, R. Hybridization in plants: Old ideas, new techniques. Plant Physiology 173, 65-78 (2017).

105 Mitchell, N. et al. Correlates of hybridization in plants. Evolution Letters 3, $570-585$ (2019).

1026106 Payseur, B. A. \& Rieseberg, L. H. A genomic perspective on hybridization and speciation. Molecular Ecology 25, 2337-2360 (2016).

107 Bodenes, C. et al. Comparative mapping in the Fagaceae and beyond with EST-SSRs. BMC Plant Biology 12 (2012).

108 Cannon, C. H. \& Petit, R. J. The oak syngameon: more than the sum of its parts. New Phytologist 226, 978-983 (2020).

109 Chen, S. C., Cannon, C. H., Kua, C. S., Liu, J. J. \& Galbraith, D. W. Genome size variation in the Fagaceae and its implications for trees. Tree Genetics \& Genomes 10, 977-988 (2014).

110 Kremer, A. et al. Genomics of Fagaceae. Tree Genetics \& Genomes 8, 583610 (2012).

111 Neale, D. B., Martinez-Garcia, P. J., De La Torre, A. R., Montanari, S. \& Wei, X. X. Novel insights into tree biology and genome evolution as revealed through genomics. Annual Review of Plant Biology 68, 457-483 (2017).

112 Staton, M. et al. Substantial genome synteny preservation among woody angiosperm species: comparative genomics of Chinese chestnut (Castanea mollissima) and plant reference genomes. BMC Genomics 16, 744 (2015).

113 Manos, P. S. \& Stanford, A. M. The historical biogeography of Fagaceae: Tracking the tertiary history of temperate and subtropical forests of the Northern Hemisphere. International Journal of Plant Sciences 162, S77-S93 (2001). 
1047114 Salojarvi, J. et al. Genome sequencing and population genomic analyses 1048 provide insights into the adaptive landscape of silver birch. Nature Genetics 49, 904-912 (2017).

1050115 Mishra, B. et al. A reference genome of the European beech (Fagus sylvatica L.). Gigascience 7, giy063 (2018).

1052116 Xing, Y. et al. Hybrid de novo genome assembly of Chinese chestnut (Castanea mollissima). Gigascience 8, giz112 (2019).

1054117 Sork, V. L. et al. High-quality genome and methylomes illustrate features 1055 underlying evolutionary success of oaks. Preprint at bioRxiv http://10.1101/2021.1104.1109.439191 (2021).

1057118 Emms, D. M. \& Kelly, S. OrthoFinder: Phylogenetic orthology inference for comparative genomics. Genome Biology 20, 238 (2019).

1059119 Camacho, C. et al. BLAST+: Architecture and applications. BMC Bioinformatics 10, 42 (2009).

1061120 Bolger, A. M., Lohse, M. \& Usadel, B. Trimmomatic: A flexible trimmer for Illumina sequence data. Bioinformatics 30, 2114-2120 (2014).

$1063121 \mathrm{Li}, \mathrm{H}$. Aligning sequence reads, clone sequences and assembly contigs with BWA-MEM. Preprint at http://arxiv.org/abs/1303.3997v2 (2013).

1065122 McKenna, A. et al. The genome analysis toolkit: A MapReduce framework for analyzing next-generation DNA sequencing data. Genome Research 20,

124 Haudry, A. et al. An atlas of over 90,000 conserved noncoding sequences 1297-1303 (2010).

1074125 Hupalo, D. \& Kern, A. D. Conservation and functional element discovery in

1077126 Dierckxsens, N., Mardulyn, P. \& Smits, G. NOVOPlasty: De novo assembly of organelle genomes from whole genome data. Nucleic Acids Research 45, e18 (2017) 

rapid, accurate, and flexible batch annotation of plastomes. Plant Methods 15, 50 (2019).

128 Kearse, M. et al. Geneious Basic: An integrated and extendable desktop software platform for the organization and analysis of sequence data. Bioinformatics 28, 1647-1649 (2012).

129 Katoh, K. \& Standley, D. M. Mafft multiple sequence alignment software version 7: Improvements in performance and usability. Molecular Biology and Evolution 30, 772-780 (2013).

130 Ronquist, F. et al. Mrbayes 3.2: Efficient Bayesian phylogenetic inference and model choice across a large model space. Systematic Biology 61, 539-542 (2012).

131 Lanfear, R., Frandsen, P. B., Wright, A. M., Senfeld, T. \& Calcott, B. Partitionfinder 2: New methods for selecting partitioned models of evolution for molecular and morphological phylogenetic analyses. Molecular Biology and Evolution 34, 772-773 (2017).

132 Akaike, H. New look at statistical-model identification. IEEE Transactions on Automatic Control 19, 716-723 (1974).

133 Zhang, C., Rabiee, M., Sayyari, E. \& Mirarab, S. ASTRAL-III: Polynomial time species tree reconstruction from partially resolved gene trees. $B M C$ Bioinformatics 19, 153 (2018).

134 Chifman, J. \& Kubatko, L. Quartet inference from SNP data under the coalescent model. Bioinformatics 30, 3317-3324 (2014).

135 PAUP*. Phylogenetic analysis using parsimony (* and other methods). v. Version 4. (Sinauer Associates, Sunderland, 2003).

136 Yang, Z. PAML 4: Phylogenetic analysis by maximum likelihood. Molecular Biology and Evolution 24, 1586-1591 (2007).

137 dos Reis, M. \& Yang, Z. Approximate likelihood calculation on a phylogeny for Bayesian estimation of divergence times. Molecular Biology and Evolution 28, 2161-2172 (2011).

138 Rabosky, D. L. et al. BAMMtools: An R package for the analysis of evolutionary dynamics on phylogenetic trees. Methods in Ecology and Evolution 5, 701-707 (2014). 
1113139 Salichos, L., Stamatakis, A. \& Rokas, A. Novel information theory-based 1114 measures for quantifying incongruence among phylogenetic trees. Molecular Biology and Evolution 31, 1261-1271 (2014).

1116140 Smith, S. A., Moore, M. J., Brown, J. W. \& Yang, Y. Analysis of 1117 phylogenomic datasets reveals conflict, concordance, and gene duplications 1118 with examples from animals and plants. BMC Evolutionary Biology 15, 150 1119 (2015)

1120141 Xia, X. H., Xie, Z., Salemi, M., Chen, L. \& Wang, Y. An index of substitution 1121 saturation and its application. Molecular Phylogenetics and Evolution 26, 1-7 $1122 \quad$ (2003)

1123142 Xia, X. DAMBE7: New and improved tools for data analysis in molecular 1124 biology and evolution. Molecular Biology and Evolution 35, 1550-1552 1125 (2018)

1126143 McInerney, J. O. GCUA: General codon usage analysis. Bioinformatics 14, 1127 372-373 (1998).

1128144 Folk, R. A., Mandel, J. R. \& Freudenstein, J. V. Ancestral gene flow and 1129 parallel organellar genome capture result in extreme phylogenomic discord in a lineage of angiosperms. Systematic Biology 66, 320-337 (2017).

146 Olave, M., Avila, L. J., Sites, J. W., Morando, M. \& Freckleton, R. Detecting

149 Durand, E. Y., Patterson, N., Reich, D. \& Slatkin, M. Testing for ancient

145 Sukumaran, J. \& Holder, M. T. DendroPy: A Python library for phylogenetic computing. Bioinformatics 26, 1569-1571 (2010). hybridization by likelihood calculation of gene tree extra lineages given explicit models. Methods in Ecology and Evolution 9, 121-133 (2017).

147 Than, C. \& Nakhleh, L. Species tree inference by minimizing deep coalescences. PLoS Computational Biology 5, e1000501 (2009).

148 Malinsky, M., Matschiner, M. \& Svardal, H. Dsuite - Fast D-statistics and related admixture evidence from VCF files. Molecular Ecology Resources 21, 584-595 (2021). admixture between closely related populations. Molecular Biology and Evolution 28, 2239-2252 (2011).

150 Green, R. E. et al. A draft sequence of the Neandertal genome. Science 328, 710-722 (2010). 
1146151 Benjamini, Y. \& Hochberg, Y. Controlling the false discovery rate: A 1147 practical and powerful approach to multiple testing. Journal of the Royal 1148 Statistical Society Series B-Methodological 57, 289-300 (1995).

1149152 Solis-Lemus, C. \& Ane, C. Inferring phylogenetic networks with maximum 1150 pseudolikelihood under incomplete lineage sorting. PLoS Genetics 12, 1151 e1005896 (2016).

1152153 Solis-Lemus, C., Bastide, P. \& Ane, C. Phylonetworks: A package for 1153 phylogenetic networks. Molecular Biology and Evolution 34, 3292-3298 $1154 \quad$ (2017).

1155154 Hejase, H. A. \& Liu, K. J. A scalability study of phylogenetic network 1156 inference methods using empirical datasets and simulations involving a single 1157 reticulation. BMC Bioinformatics 17, 422 (2016).

1158155 Shen, X. X., Hittinger, C. T. \& Rokas, A. Contentious relationships in 1159 phylogenomic studies can be driven by a handful of genes. Nature Ecology \& $1160 \quad$ Evolution 1, 126 (2017).

1161156 DePristo, M. A. et al. A framework for variation discovery and genotyping 1162 using next-generation DNA sequencing data. Nature Genetics 43, 491-498 1163 (2011).

1164157 Browning, B. L. \& Browning, S. R. Improving the accuracy and efficiency of 1165 identity-by-descent detection in population data. Genetics 194, 459-471 1166 (2013).

\section{Acknowledgements}

1170 We thank Al Keuter for assistance with the layout design and select photos of cupules 1171 used in in Figure 1. This work was supported by the Guangdong Natural Science

1172 Funds for Distinguished Young Scholar (grant no. 2018B030306040 to B.W.),

1173 National Natural Science Foundation of China (grant no. NSFC 31971673 to B.W.),

1174 and US National Science Foundation (grant no. 1146102 to P.S.M.)

\section{Data availability statement}


1176 Short reads of whole genome sequencing data have been submitted to NCBI 1177 (BioProject number $\mathrm{xxx}$ ). Alignments of nuclear genes and plastomes are archived in 1178 the Dryad digital data repository (xx).

1179 Author contributions

1180 B.-F.Z., S.Y., M.K., P.S.M. and B.W. designed the project. B.-F.Z., S.Y., Y.-Y.L., 1181 Y.S., X.-Y.C. and Q.-Q.A. collected data. B.-F.Z., S.Y., A.A.C., Y.-Y.L., P.S.M. and 1182 B.W. analyzed data. B.-F.Z., S.Y., A.A.C., P.S.M. and B.W. wrote the paper. All 1183 authors read and approved the paper.

1184

\section{Competing interests statement}

1186 The authors declare no competing interests. 


\section{Fig. 1}

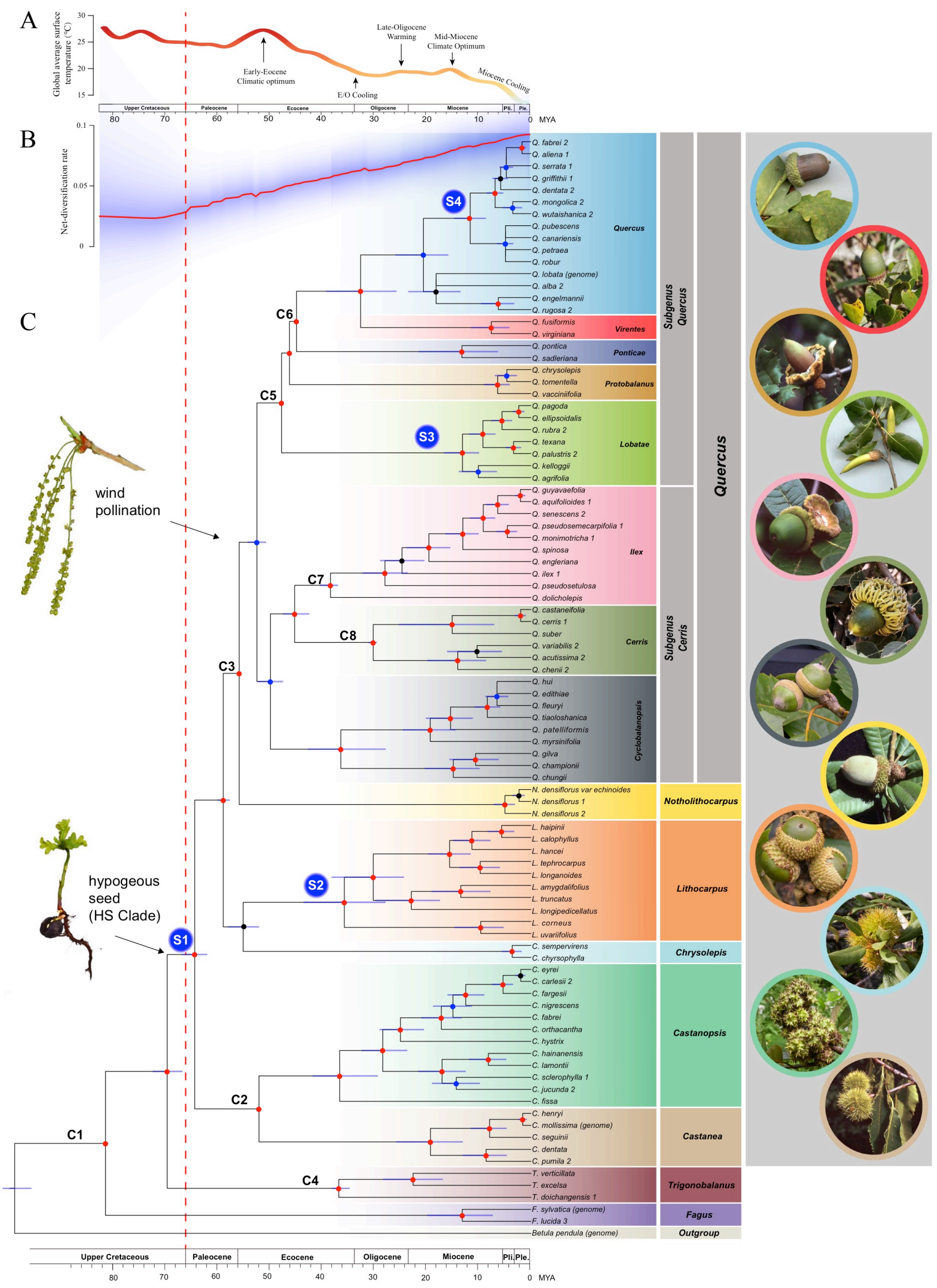


Fig. 1 Phylogenetic relationships and divergence time estimation of Fagaceae inferred from analyses of 2124 nuclear genes. (A) The global climate curve during the last 82 million years (modified from Tierney et al. 2020). Major climate events were indicated. (B) Rate-through-time plot showing the net diversification rate (species/ million years) of Fagaceae. Red line is the median and the blue shadow represents the 95\% confidence interval. (C) Chronogram derived from ASTRAL-III tree based on concatenated nuclear data. Nodes showing consistent relationships between ASTRALIII, SVDquartets, maximum likelihood (ML), and MrBayes are marked with red (phylogenetic support $\geq 95$ in all four analyses) and blue (support $<95 \%$ in any one of the four analyses). Nodes showing conflicting relationships among analyses are marked with black dots. Light blue bars on nodes represent 95\% confidence intervals of divergence time estimates and dashed vertical red line represents the age of the Cretaceous-Paleogene boundary (66 million years ago). Geological timescale is shown at bottom. Fossil calibration nodes are indicated with $\mathrm{C} 1-\mathrm{C} 8$ (stem calibration node; Table S3). S1- S4 indicate four nodes where shifts in diversification rate were identified. Taxonomic labels of genera, subgenera and sections follow Manos et al. 2001, Manos et al. 2008; and Denk et al. 2017. Illustrations: lax catkins indicate the placement of the change from insect-pollination to wind-pollination that diagnoses the genus Quercus; hypogeous seed and seedling marks the origin of the HS clade. Images: representative cupule types are shown on the right. A consistent color scheme was used for taxonomic labels and image borders. 


\section{Fig. 2}

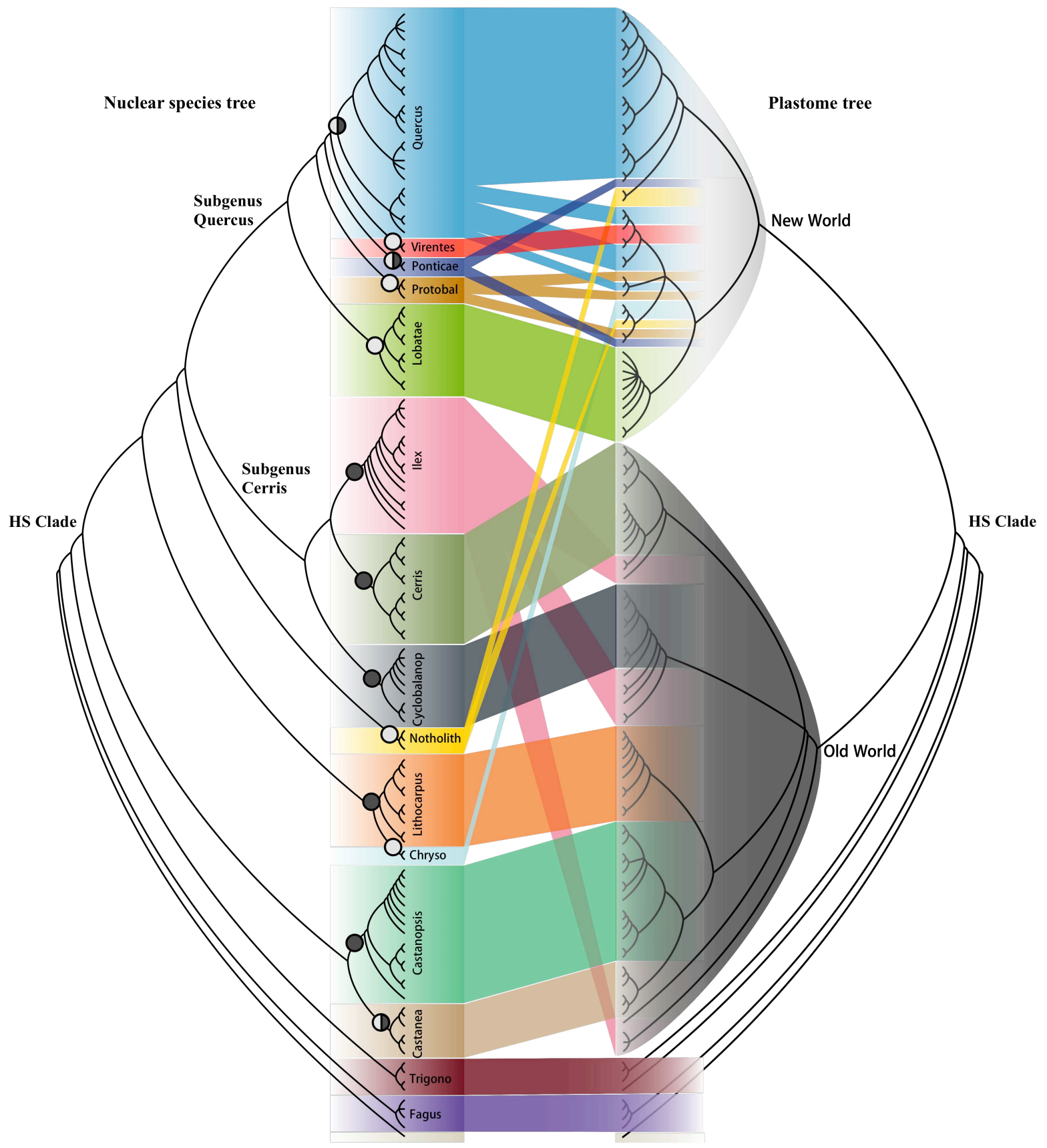

Fig. 2 Conflicts between nuclear (left) and plastome (right) species trees. Pie charts on nodes indicate the geographic distribution of the clade (black $=$ Old World, white $=$ New World). The HS clade consists of six genera divided into two major plastome clades: New World (light grey) and Old World (dark grey). Lineage colors are consistent with the color scheme in Fig. 1. Abbreviations: Protobal, section Protobalanus; Cyclobalanop, section Cyclobalanopsis; Notholith, Notholithocarpus; Chryso, Chrysolepis; Trigono, Trigonobalanus. 


\section{Fig. 3}

A

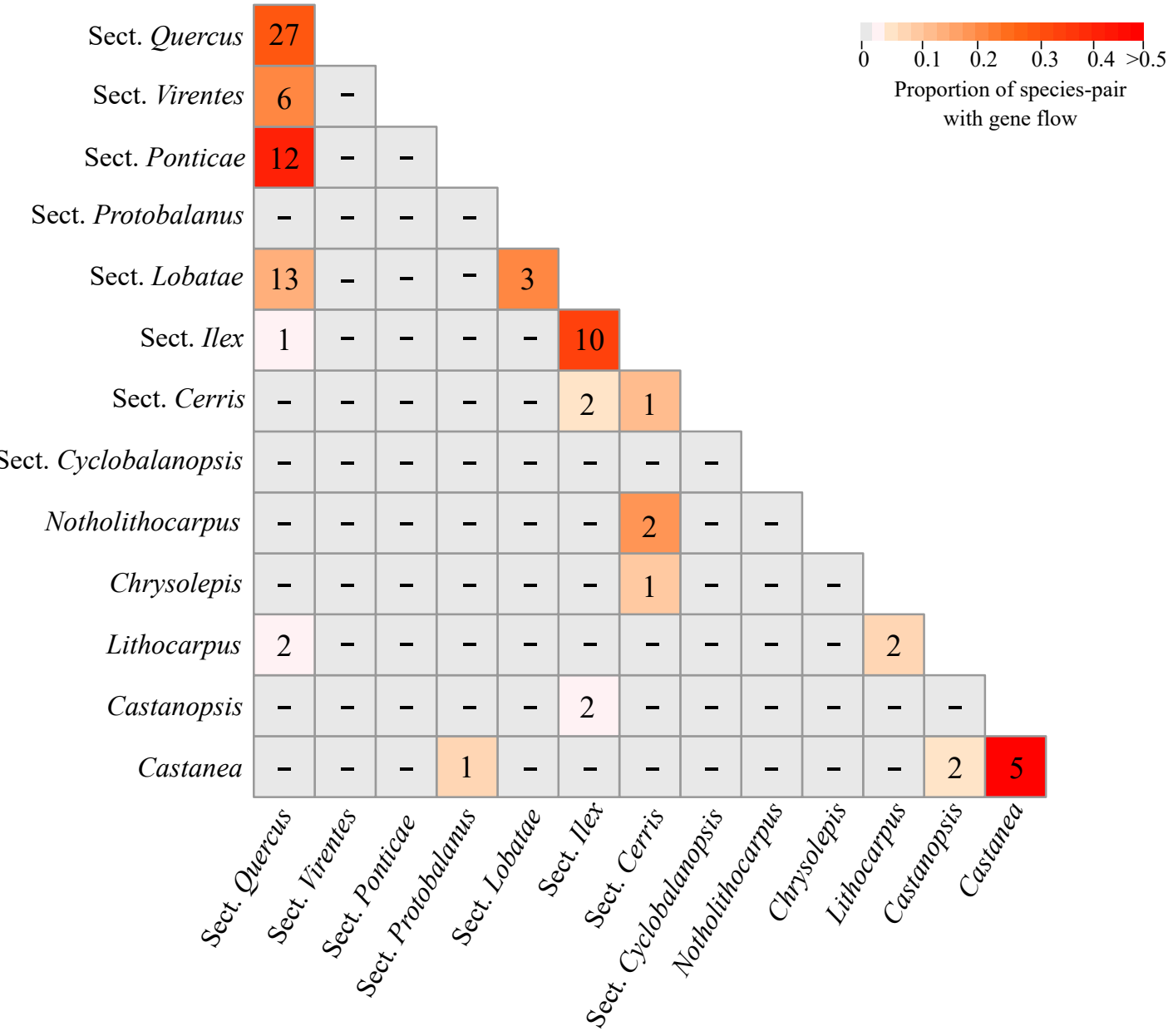

B
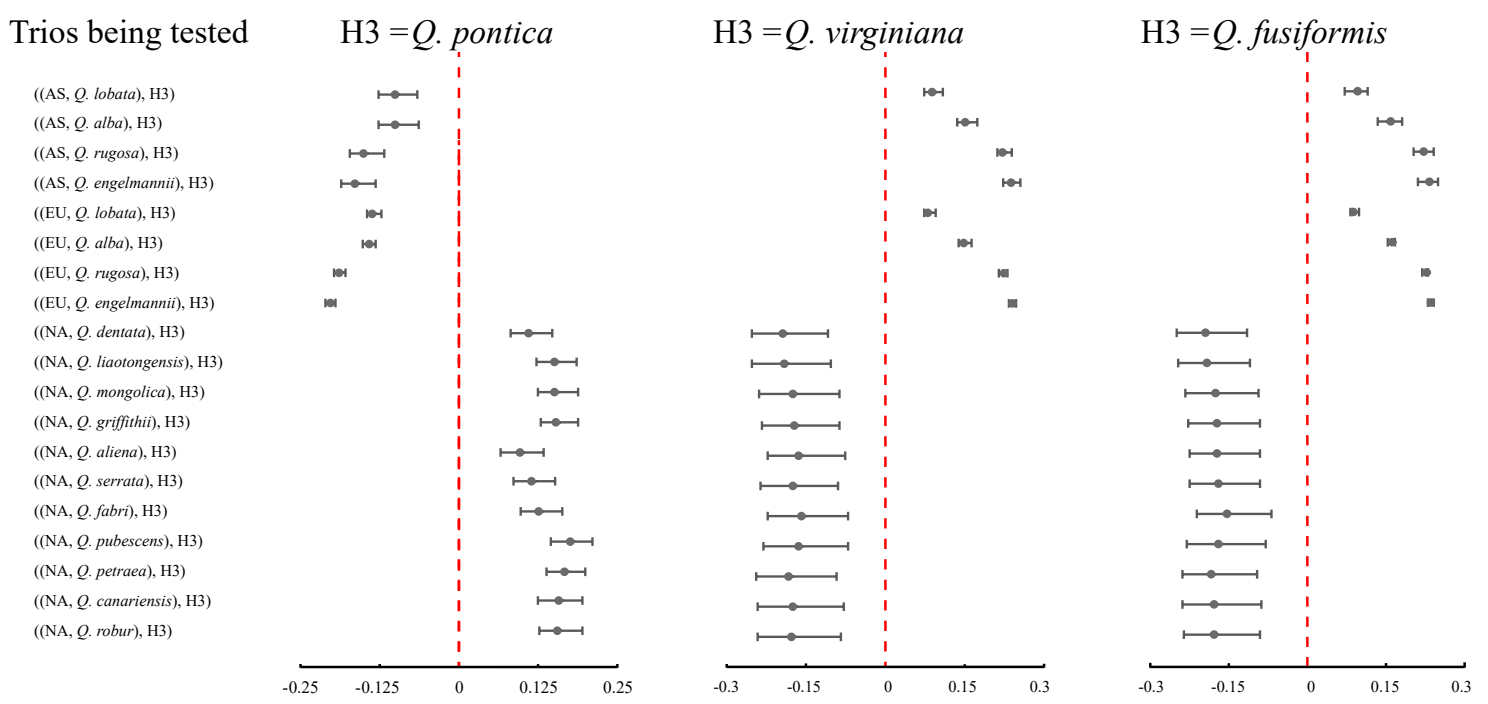
Fig. 3 Gene flow between Fagaceae species revealed by $D$-statistic test. (A) Number of species-pairs with significant $D$ value $(P<0.01$ after Bonferroni correction) between sections of Quercus and other genera. Numbers on diagonal line indicate gene flow within each section or genus. Cells are colored based on the ratio of species-pairs with gene flow, with warmer colors indicating a higher proportion of species-pairs showing gene flow. For example, significant gene flow was detected for 12 species-pairs between sections Quercus (white oak) and Ponticae, representing $41 \%$ of tested species-pairs between these two sections. (B) Distribution of $D$ values for white oaks vs. $Q$. pontica (left), and two species of section Virentes, Q. virginiana (middle) and Q. fusformis (right). Each line summarizes a set of $D$-statistic tests performed on trios in the format $((\mathrm{H} 1, \mathrm{H} 2), \mathrm{H} 3)$ with different $\mathrm{H} 1$ species and fixed $\mathrm{H} 2$ and $\mathrm{H} 3$ species (one of the three species above). Both $\mathrm{H} 1$ and $\mathrm{H} 2$ were white oaks, but represent different lineages. For example, if $\mathrm{H} 2$ was a North American white oak, then H1 was sampled from European or Asian white oaks. In each panel, points represent mean $D$ values and error bars represent minimum and maximum $D$ values across multiple tests. $\mathrm{EU}=$ European white oak; AS = Asian white oak; NA = North American white oak. A negative $D$ value indicates gene flow between $\mathrm{H} 1$ and $\mathrm{H} 3$ while a positive $D$ value indicates gene flow between the $\mathrm{H} 2$ taxon and H3. Quercus pontica shows a clear pattern of gene flow with EU and AS white oaks but not with NA white oaks while the opposite pattern is recovered for $Q$. virginiana and $Q$. fusiformis. 


\section{Fig. 4}

A

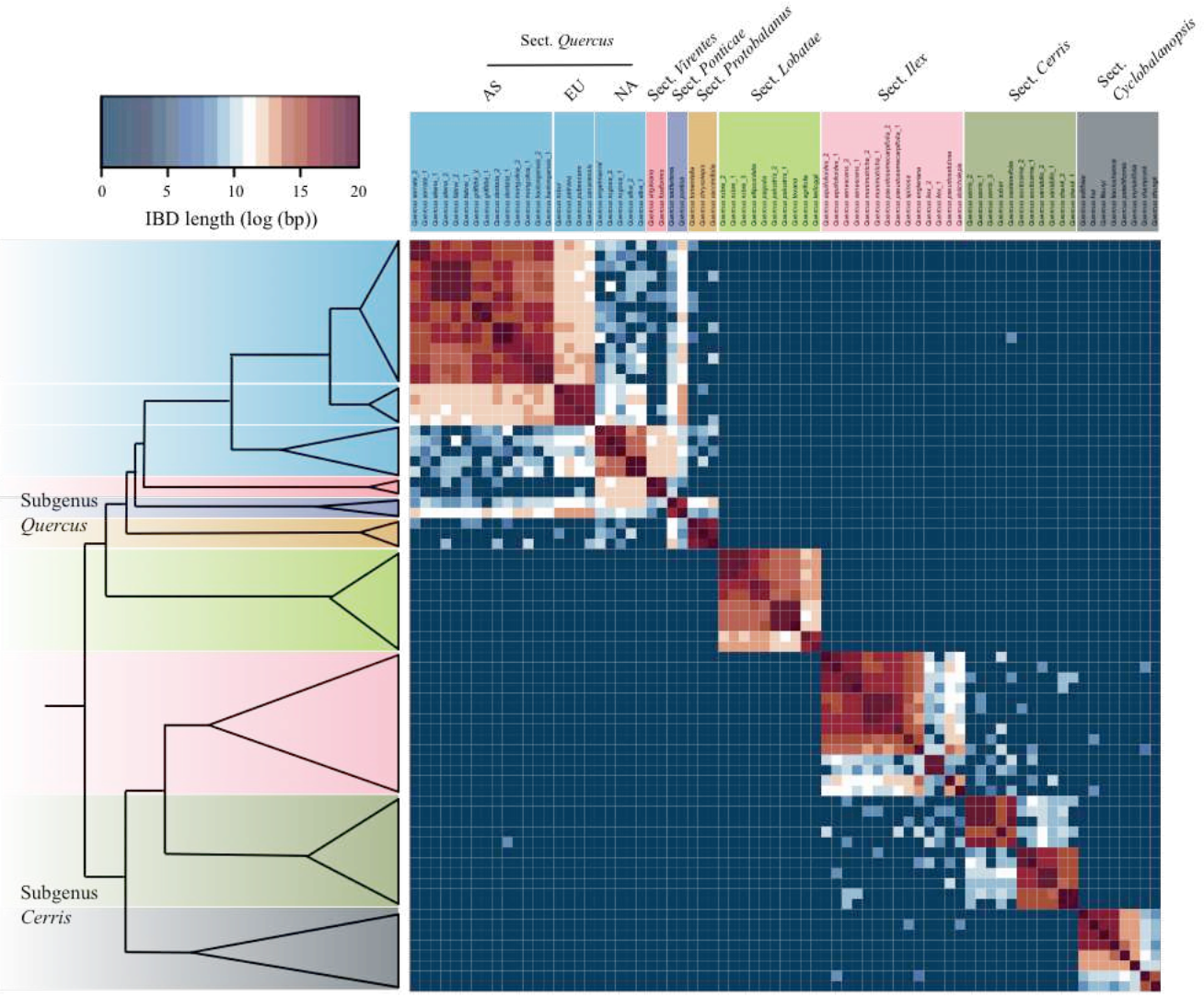

$\mathrm{B}$

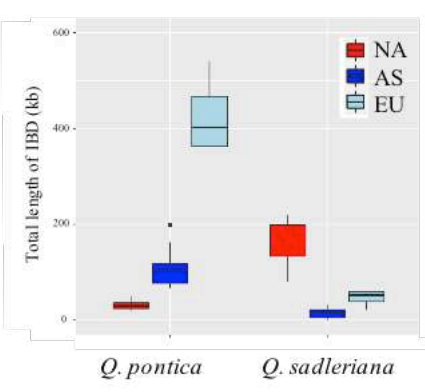

$\mathrm{C}$

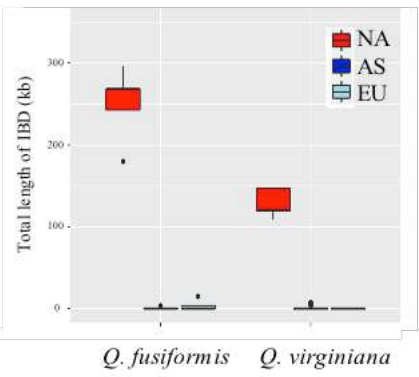

$\mathrm{D}$

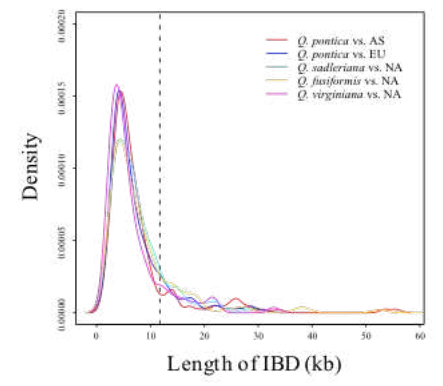

Fig. 4 Shared IBD blocks between Quercus species. (A) Heatmap indicating the total length of IBD blocks for each pair of comparisons. (B) and (C) box plots show shared total length of IBDs between sections Ponticae and Quercus, and between sections Virentes and Quercus. NA = North American white oak; EU = European white oak; AS = Asian white oak. (D) Kernel distribution of the length of shared IBD blocks between sections. Vertical black line (at $11724 \mathrm{bp}$ ) indicates the length at which IBD blocks are significantly longer than the expectation for selectively neutral introgressed fragments maintained in a population under a constant recombination rate of $10^{-8}$ per site per year, assuming an average divergence time of 3 million years $(P<0.05$ after Bonferroni correction; see details in Methods). 


\section{Supplementary Files}

This is a list of supplementary files associated with this preprint. Click to download.

- SupplementaryInformation.pdf 